\title{
Damage detection methodology on beam-like structures based on combined modal Wavelet Transform strategy
}

\author{
Roger Serra ${ }^{1, *}$ and Lautaro Lopez ${ }^{1,2}$ \\ ${ }^{1}$ INSA Centre Val de Loire Laboratoire de Mécanique et Rhéologie, 3 rue de la Chocolaterie, 41000 Blois, France \\ ${ }^{2}$ Universidad Nacional de Rosario (UNR), Facultad de Ciencias Exactas, Ingeniería y Agrimensura, Rosario, Argentina
}

Received: 12 December 2016 / Accepted: 16 January 2018

\begin{abstract}
Different approaches on the detection of damages based on dynamic measurement of structures have appeared in the last decades. They were based, amongst others, on changes in natural frequencies, modal curvatures, strain energy or flexibility. Wavelet analysis has also been used to detect the abnormalities on modal shapes induced by damages. However the majority of previous work was made with non-corrupted by noise signals. Moreover, the damage influence for each mode shape was studied separately. This paper proposes a new methodology based on combined modal wavelet transform strategy to cope with noisy signals, while at the same time, able to extract the relevant information from each mode shape. The proposed methodology will be then compared with the most frequently used and wide-studied methods from the bibliography. To evaluate the performance of each method, their capacity to detect and localize damage will be analyzed in different cases. The comparison will be done by simulating the oscillations of a cantilever steel beam with and without defect as a numerical case. The proposed methodology proved to outperform classical methods in terms of noisy signals.
\end{abstract}

Keywords: Damage assessment / modal analysis / wavelet transform / damage indicator / cantilever beam

\section{Introduction}

Damages such as cracks and pores are inevitable in aerospace, aeronautical, mechanical and civil engineering structures during their service life. The study of damages in structures is an important prospect to evaluate in order to ensure their safety and prevent collapses. Some structures, such as large bridges, are required to be continuously evaluated to detect possible damages and repair them as soon as possible to never stop their service. Moreover, such damage identifications have significant life-safety implications.

Structural health monitoring (SHM) is an efficient way to evaluate the state of a structure. Consequently, it guarantees safety and efficiency of structures throughout their life. This process involves the measurement of a structure over time, the extraction of damage-sensitive features from the measurements and their analysis to determine the current state of the system. Assessment methods should be able to diagnose damages at an early stage, be non-destructive and low-cost. Many methods were proposed along history to identify problems in structures. Visual inspection was one of the first to appear,

\footnotetext{
* email: roger.serra@insa-cvl.fr
}

however detecting cracks is not always possible with this method since some zones of the structure might be restricted to sight. Nowadays, cracks are generally being detected by non-destructive testing methods (ultrasonic testing, radiographic, X-ray, eddy-current etc.). Vibrationbased structural health monitoring is one of them based on the fact that a loss of stiffness provoked by damages affects the dynamic response of the structure. It is mainly attractive because of its ability to monitor and detect damage from a global testing of the structure. Thus, it does not require accessibility and measuring of potentially damaged areas.

In this paper, a new approach for damage identification based on combined modal Wavelet Transform strategy is proposed in which the drawback of Cao and Quiao [1] methodology are dealt with the modal processing presented in Solis et al. [2]. The result is a noise-robustness approach that makes use of modal data in a more efficient way. First, extracted or simulated modal shapes of a damaged beam are refined in order to reduce random noise and interferences. The difference between undamaged modal shapes and the refined ones are weighted depending on the changes in corresponding natural frequencies. Finally, the addition of the coefficients of Continuous Wavelet Transform (CWT) for each difference in modal shapes is used to localize damages. 
Wavelet background theory is firstly presented in this article. Then the proposed methodology is described. Several numerical examples are provided in order to compare some of the classical methods with the proposed one. The numerical data, which involve the calculation of the first few natural frequencies and modal shapes from a cracked Euler-Bernoulli beam, are obtained with a commercial software COMSOL. Nine cases with different crack depth and positions are studied. The influence of noise in modal shapes is also presented. To sum up, conclusions are drawn.

\section{Literature review}

The first approach of using modal analysis for damage detection relied on that changes in the properties of structures modify their eigen frequencies of vibration. Cawley and Adams [3] were one of the first to develop a method based on the shift of more than one frequency that could yield to the location of the damage. Salawu [4] presented a review on the use of natural frequency changes for damage detection. Serra et al. [5], and more recently Gautier et al. [6] have studied the performance of subspace fitting methods to detect and locate damages in a structure (G. Gautier R. S.-M.) [7] and roller bearings [8].

The change in natural frequencies is not considerable when the damage is small, which makes preventive maintenance difficult to carry out. As a result, damage indicators based on vibrational modal shapes were introduced. They were mainly attractive because the change in stiffness provoked by the damage is reflected locally in the modal shapes. Allemang and Brown [9] presented probably the first use of mode shape information for damage detection in a structure. Modal Assurance Criterion was used to determine if a body flap was damaged or not. In Kim et al. [10], a comparison between frequencybased damage detection methods and modal shape-based detection methods was carried out. The authors formulated algorithms for both methods to locate damage and estimate the size of the simulated crack. They concluded that changes in modal shapes are much more sensitive compared to changes in natural frequencies. Coordinate Modal Assurance Criterion was introduced by Lieven and Ewins [11], which is conceptually similar to MAC but it tries to solve the problem of location of the damage. Pandey et al. [12] introduced the concept of mode shape curvature for damage localization. In their study, both a cantilever and a simply supported beam model were used to demonstrate the effectiveness of changes in modal curvature as damage indicator to detect and localize damage. Wahab and De Roeck [13] found that the modal curvature of lower frequency vibration modes was in general more accurate than those of the higher frequency vibration modes. The advantage of the curvature mode shape over the displacement mode shape in identifying the location of damage was demonstrated by Salawu and Williams [14]. Based on the curvature method, Stubbs et al. [15] proposed the Damage Index Method, which is the comparison between square curvatures of the structure before and after the damage. An approach based on the use of strain energy to detect and localize damage was presented by Sazonov and Klinkhachorn [16]. The main drawback of the mentioned methods is the need to calculate derivatives numerically. The differential operator entails numerical error that might be bounded but not negligible. Pandey and Biswas [17] proposed the use of changes in the dynamically measured flexibility matrix to detect and localize damage. They showed that the flexibility matrix of a structure can be easily and accurately estimated from a few low frequency vibration modes of the structure.

Another important approach for damage detection is based on Wavelet Transform. The Wavelet Transform is a mathematical tool that decomposes a signal into a localized time (or scale) and frequency (or scale) domain, as Daubechies [18] explained. Wavelets are purposefully crafted to have specific properties that make them useful for signal processing. As a matter of fact, Wavelet Analysis is capable of revealing "hidden" aspects of the input signal, which in case of SHM are provoked by damages. The main advantages of Wavelet Analysis is the ability to perform local analysis of signals, its noise robustness and the possibility to assess the state of a structure without the baseline undamaged state of the structure. The first publication considered to mention Wavelet Analysis as damage indicator is Surace and Ruotolo in 1994 [19]. Liew and Wang [20] proposed a damage detection method based on the sudden changes in the variation of Wavelet Transform coefficients. Hong et al. [21] and Douka et al. [22] used Lipschitz exponent to localize and estimate the size of a crack. They came to the conclusion that the size of the crack can easily be determined by the coefficients of wavelet transform. Their results were confirmed both numerically and experimentally. Chen et al. [23] used Lipschitz exponent for the same reason and proved its efficiency with different types of excitation. They also analyzed the influence of damage location and mother wavelets as well as noise in signals. $\mathrm{Wu}$ and Wang [24] used high resolution laser with Continuous Wavelet Transform (CWT) to measure the deflection profile of a cracked cantilever beam to locate its damage. Argoul and Le [25] have presented an instantaneous indicator based on continuous cauchy wavelet analysis. Solis et al. [2] presented a method based on the addition of the CWT coefficients for different weighted mode shapes according to the corresponding variation of the changes in natural frequencies for each one to compute an overall result along the structure. However, in their laboratory work they had to apply several impacts in order to obtain 65 measurement points and reduce the experimental noise. This methodology can become expensive and not practical in real cases scenarios.

Discrete Wavelet Transform (DWT) takes into account much less situations to calculate coefficients but the analysis will be more efficient in terms of calculation work. Naldi and Venini [26] in 1997 and Lu and Hsu [27] in 1999 were one of the first to use Discrete Wavelet Analysis to detect and localize damage. They analyzed a 1-D structure and flexible strings respectively and came to the conclusion that this method is capable 
to correctly assess the state of a structure using its dynamics characteristics. Even though DWT is widely used in structural and machine health monitoring, it is a non-redundant decomposition analysis, as Mallat [28] explains. This provokes DWT to be shift-variant. In a shift-invariant system, the contemporaneous response of the output variable to a given value of the input variable does not depend on when it occurs. This feature is very important for monitoring systems. To cope with this issue, Stationary Wavelet Transform (SWT) was introduced which makes the wavelet transform redundant. A comparison between DWT and SWT is shown in Zhong and Oyadiji [29] where the authors concluded that SWT is much more suitable for damage detection than DWT.

The majority of the studies were numerical. On the other hand, in the experimental ones like Rucka and Wilde [30], a great amount of sensors had to be used to deal with random interferences in the measured data and noise. A technique called Integrated Wavelet Analysis, which combines the advantages of SWT and CWT, was proposed by Cao and Quiao [1]. Their method implements SWT to refine the modal shapes and then look for damage applying CWT to the refined modal shape. Masoumi and Ashory [31] made numerical and experimental studies on this method to localize cracks. However, they only took into account the first modal shape in their calculations. It is well known that only one mode shape is not enough for damage detection since the sensitivity of the mode depends on the location of the damage.

\section{Wavelet background theory}

Both the Fourier and Wavelet Transform measure similarity between a signal and an analyzing function. The two transforms differ in their choice of analyzing function. In the Fourier transform, the analyzing functions are complex exponentials $e^{j w x}$ where $x$ is the independent space variable. In Wavelet Transform, the analyzing function is a wavelet $\psi$. Wavelets are purposefully crafted to have specific properties that make them useful for signal processing. Daubechies [17] and Mallat [32] developed the principal elements of Wavelet Transform Theory.

A mother wavelet $\psi(x)$ can be defined as an oscillating function with zero average value:

$$
\int_{-\infty}^{+\infty} \psi(x) \mathrm{dx}=0
$$

The function $\psi(\mathrm{x})$ is used to create a family of wave$\operatorname{lets} \psi_{\mathrm{a}, \mathrm{b}}(x)$ :

$$
\psi_{\mathrm{a}, \mathrm{b}}(x)=\frac{1}{\sqrt{\mathrm{a}}} \psi\left(\frac{x-\mathrm{b}}{\mathrm{a}}\right)
$$

Where real numbers $a$ and $b$ are dilation and translation parameters, respectively.

\subsection{Continuous Wavelet Transform (CWT)}

The CWT compares the input signal $f(x)$ to shifted and compressed or stretched versions of a mother wavelet $\psi(\mathrm{x})$. Stretching or compressing a function corresponds to the notion of scale. By comparing the signal to the wavelet at various scales and positions, a function of two variables is obtained.

For a scale parameter $a>0$ and position $b$, the CWT is defined as:

$$
C W T_{f}(a, b)=\frac{1}{\sqrt{a}} \int_{-\infty}^{+\infty} f(x) \psi^{*}\left(\frac{x-b}{a}\right) d x
$$

where $\psi^{*}$ denotes the conjugate of the mother wavelet. By varying the values of the scale parameter $a$, and the position parameter $b$, CWT coefficients $C W T_{f}(a, b)$ are obtained. Regarding the space-scale view of signals, it is worth analyzing the ability of wavelet to react to subtle changes or discontinuities in signals. An important feature of a wavelet is its number of vanishing moments. If a wavelet has $n$ vanishing moments, then:

$$
\int_{-\infty}^{+\infty} x^{k} \psi(x) d x=0 \text { for } k=0 \ldots n-1
$$

For any polynomial of smaller order than the number of vanishing moments, the wavelet will give null values. The number of vanishing moments indicates how sensitive the wavelet is to low order signals, and can be chosen to take into account only certain components of the signal.

It can be mathematically demonstrated [32] that for a wavelet with $n$ vanishing moments and a fast decay there exists a function $\theta(x)$ called smoothing function defined as follows:

$$
\psi(\mathrm{x})=\frac{d^{n} \theta(\mathrm{x})}{d x^{n}}, \int_{-\infty}^{+\infty} \theta(\mathrm{t}) \mathrm{dt} \neq 0
$$

Therefore, a wavelet with $n$ vanishing moments can be rewritten as the $n^{\text {th }}$ order derivative of the smoothing function $\theta(x)$. Continuous Wavelet Transform coefficients can be expressed as:

$$
\begin{aligned}
C W T_{f}(a, b) & =\int_{-\infty}^{+\infty} f(x) \psi^{*}\left(\frac{x-b}{a}\right) d x \\
& =\frac{1}{\sqrt{a}} \int_{-\infty}^{+\infty} f(x) \frac{d^{n} \theta}{d x^{n}}\left(\frac{x-b}{a}\right) d x \\
& =\frac{a^{n}}{\sqrt{a}} \int_{-\infty}^{+\infty} \frac{d^{n}}{d x^{n}}(f(x)) \theta\left(\frac{x-b}{a}\right) d x
\end{aligned}
$$

The wavelet transform described previously depends on the $n^{\text {th }}$ derivative of the signal function $f(x)$ smoothed by the function $\theta(x)$ at the scale $a$. Then singularity in a signal function $f(x)$ can be detected finding the abscissa where the maxima of the wavelet transform modulus converge at fine scales [32]. In the analyzed case, the transition in $f(x)$ will be caused by a crack and this precisely is the proposed identification method. 


\subsection{Discrete and Stationary Wavelet Analysis (DWT and SWT)}

Discrete Wavelet Analysis will result in less amount of information since it is going to be performed only on a discrete grid of parameters of dilatation and translation. An arbitrary signal $f(x)$ can be written using an orthonormal wavelet basis:

$$
f(x)=\sum_{j} \sum_{k} \mathrm{~d}_{j, k} \psi_{j, k}(x)
$$

where the coefficients are given by:

$$
\mathrm{d}_{j, k}=\int_{-\infty}^{+\infty} f(x) \psi_{j, k}(x) d x
$$

The orthonormal basis functions are generated by dilating and translating an orthonormal mother wavelet $\psi(\mathrm{x})$ :

$$
\psi_{j, k}(x)=2^{-j / 2} \psi\left(2^{-j} x-k\right)
$$

where $j$ and $k$ are the dilation and translation parameters, respectively. The contribution of the signal at level $j$ is given by:

$$
\mathrm{d}_{j}(x)=\sum_{k=1}^{n} \mathrm{~d}_{j, k} \psi_{j, k}(x) d x
$$

These coefficients are called detailed coefficients and provide information on the space behavior of the signal at different scales. Mallat [32] developed a computationally efficient method to calculate equations (7) and (8) from finite signals, which is called as Multi Resolution Analysis (MRA). A multiresolution decomposition of a signal is based on successive decomposition into approximation and detailed coefficients. In order to implement fast wavelet transform to perform MRA, DWT and SWT make use of mother wavelets as well as scaling functions and approximate coefficients, which are related to the mother wavelet. The scaling function $\varphi(x)$ is translated and dilated as follow:

$$
\phi_{j, k}(x)=2^{-j / 2} \phi\left(2^{-j} x-k\right)
$$

Both functions satisfy the two-scale equation (quadrature mirror filters):

$$
\begin{aligned}
& 2^{-j / 2} \psi\left(\frac{\mathrm{x}}{2}-k\right)=\sum_{n=-\infty}^{+\infty} g(n-2 k) \phi(x-n) \\
& 2^{-j / 2} \phi\left(\frac{\mathrm{x}}{2}-k\right)=\sum_{n=-\infty}^{+\infty} h(n-2 k) \phi(x-n)
\end{aligned}
$$

Where $\left\{h_{n}\right\}$ and $\left\{g_{n}\right\}$ are the impulse responses of low-pass and high-pass filters, respectively.

The approximate and detailed coefficients at level $j$ can be calculated from the apprimate coefficients at level $j+1$ :

$$
\begin{aligned}
& a_{j, k}=\sum_{n} h(n-2 k) a_{j+1, n} \\
& d_{j, k}=\sum_{n} g(n-2 k) a_{j+1, n}
\end{aligned}
$$

Concerning the definitions already done, a signal $f(x)$. can be decomposed up to the $J$ th level as:

$$
f(x)=\sum_{k} a_{j, k}(k) \phi_{j, k}(x)+\sum_{j=1}^{J} \sum_{k} d_{j, k}(k) \psi_{j, k}(x)
$$

Approximation coefficients have information about the identity of the signal, while detailed coefficients give information about local discontinuities. DWT is able to split a multicomponent signal into a group of component signals. Measured signals are typically a mixture of noise components with damage-related information. In this regard, the extraction of damage-related component of the signal would be useful.

As it was stated, DWT is a non-redundant decomposition which is a serious drawback for damage detection. The non-redundancy of DWT is caused by the decimation operation at each level of decomposition, which modifies the number of sampling points for each level. This drawback can be solved by using SWT, which is similar to DWT except that the number of wavelet coefficients at each level remains equal to the number of sampling points while when using DWT the reduction of the number of coefficients is $n / 2^{j}$. SWT doubles the number of input samples at each iteration. Since SWT also has the capability of decomposing a multicomponent signal, it is going to be used to refine the modal shapes and obtain the damage-related information.

\subsection{Wavelet families and properties}

There are many different admissible mother wavelets that can be used in wavelet analysis. The election of the mother wavelet depends on the signal which is going to be tested. It is worth remembering that not only the value of the scale and position affects the value of wavelet coefficients but also the choice of the wavelet. This choice should be based on a mathematical analysis of the nature of the signal. However this analysis is not always clear and the wavelet family is usually determined by trial and error. The lack of theoretical investigation on the choice of the optimal mother wavelet family is a problem since the effectiveness of the application is directly implicated to the wavelet family.

Some authors, like Ovanesova and Suárez [33] and Cao and Quiao [1] have briefly studied the most important wavelet properties. In the case of Stationary Wavelet Analysis (SWT), the mother wavelet should also have an explicit scaling function. Ovanesova and Suárez [33], Cao and Qiao [1] and Prasad et al. [34] took the characteristics previously described and concluded that reverse biorthogonal wavelets are the most appropriate since they enjoy merits as orthogonality, short size of support and symmetry. After this exigency, orthogonal and biorthogonal wavelets still remain. 

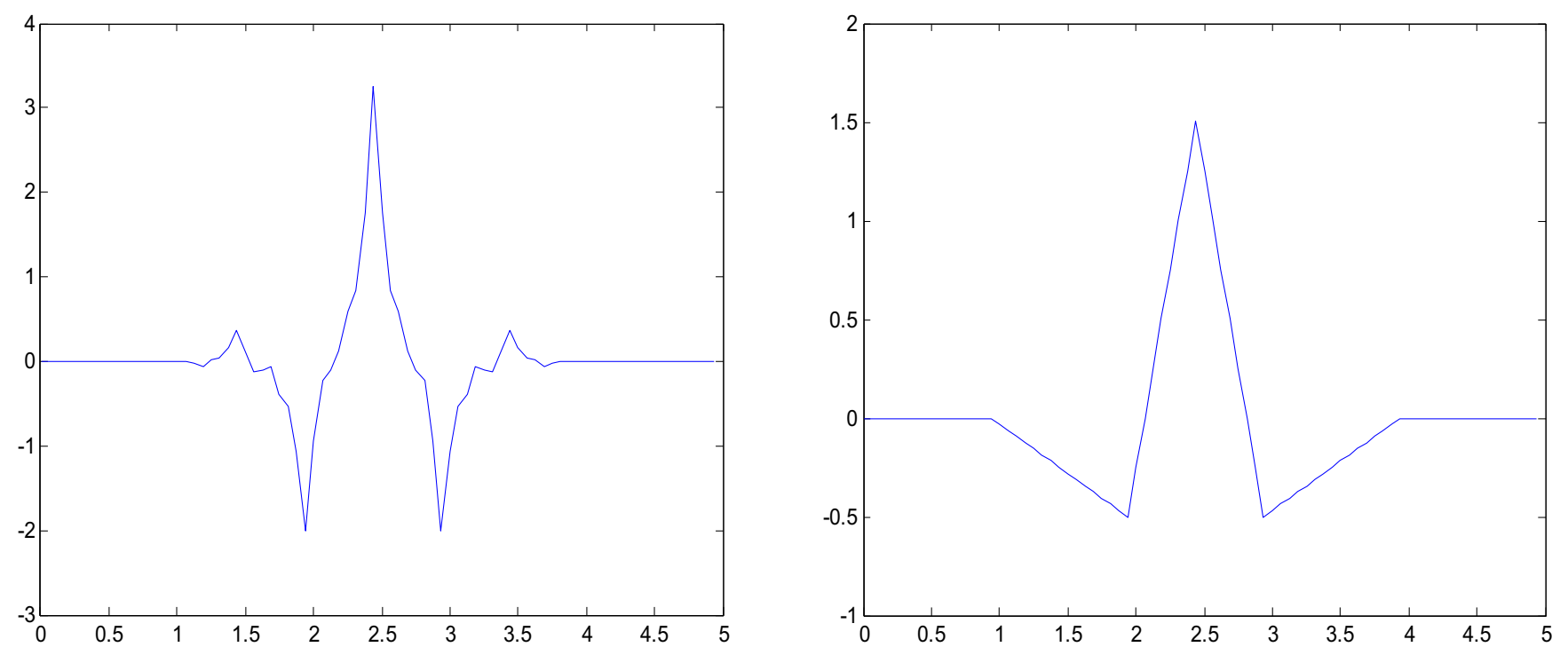

Fig. 1. rbio2.2 wavelet: decomposition (left) and reconstruction (right).

As mentioned above, the number of vanishing moments is critical for spatial detection of singularities in wavelet analysis. Cao and Qiao [1] demonstrated that even number of vanishing moments are more efficient in showing signal discontinuities. Therefore, if a wavelet with 2 vanishing modes is used [35], the CWT coefficients of mode shapes give information about the change in the second derivatives of mode shapes (modal curvature). It is well known that changes in curvatures is an efficient damage indicator. Another way to cope with the number of vanishing moments is to identify that the first modal shapes can be approximated using polynomials up to degree not less than 2 but no more than 4 . Thus, the number of vanishing moments of the optimal mother wavelet should be between 3 and 5 . An observation should be made in this regard. The first displacement modal shapes are considered to be the most accepted for damage detection since they are stable and easy to measure. However, new measurement techniques and equipment are constantly developed. Rucka [36] used a scanning laser vibrometer and concluded that high vibration modes are the most sensitive to damage detection. Since the higher vibration modes require higher polynomials to approximate them, the number of vanishing modes should also increase. Douka et al. [21] stated that wavelet having high number of vanishing moments have more stable performance.

On the other hand, wavelet with more vanishing moments have longer support sizes, which induce wider duration of peaks. This effect worsens the crack location, in other words, localization worsens as support length increases. A compromise between the number of vanishing moments and support length has to be made. For all the reasons mentioned and after several tests, it was concluded that the optimal number of vanishing moment for multiresolution analysis is 4 and for damage detection is 2: Overall, in this work, "rbio4.4" is chosen as the mother wavelet for SWT and "rbio2.2" for CWT (Figs. 1 and 2).

\section{Combined Wavelet Transform strategy for damage identification}

In this section, the proposed methodology for damage identification is described in four clear steps once the data are already obtained in laboratory conditions or by field measurements if possible. As others vibration-based damage indicators listed in the literature review, the methodology requires information about the undamaged and damaged states of the structure. An alternative, when experimental data is inaccessible, is to perform numerical simulations and simulate environmental conditions adding noise on the response. The proposed methodology is focused on how to deal with modal parameters i.e. modal shapes and natural frequencies obtained previously with appropriate methods (Fig. 3).

\subsection{Extension of modal shapes}

The Wavelet Transform is defined for an infinite interval. On the other hand, modal shapes signals are spatial thus they are defined over a finite interval. When performing Wavelet Transform, this issue leads to wrong behavior at the beginning and at the end of the signal. There exist local changes in the mentioned points since the signal starts and finishes, however those discontinuities are not caused by damage. Damages close to the beginning and end of the structure can be masked due to the high values of the coefficients near the edges.

Different approaches to this problem have been proposed in the literature, like advanced mathematical methods in Messina and Gentile [37] or more simple ones like smooth extensions of the signal from beyond the boundaries (Rucka and Wilde [29], Solis et al. [2]). By equation (2) it is possible to conclude that higher scales of CWT use more points to evaluate the signal, hence, the length of the extension of the signal depends on the scales used in the transform. 

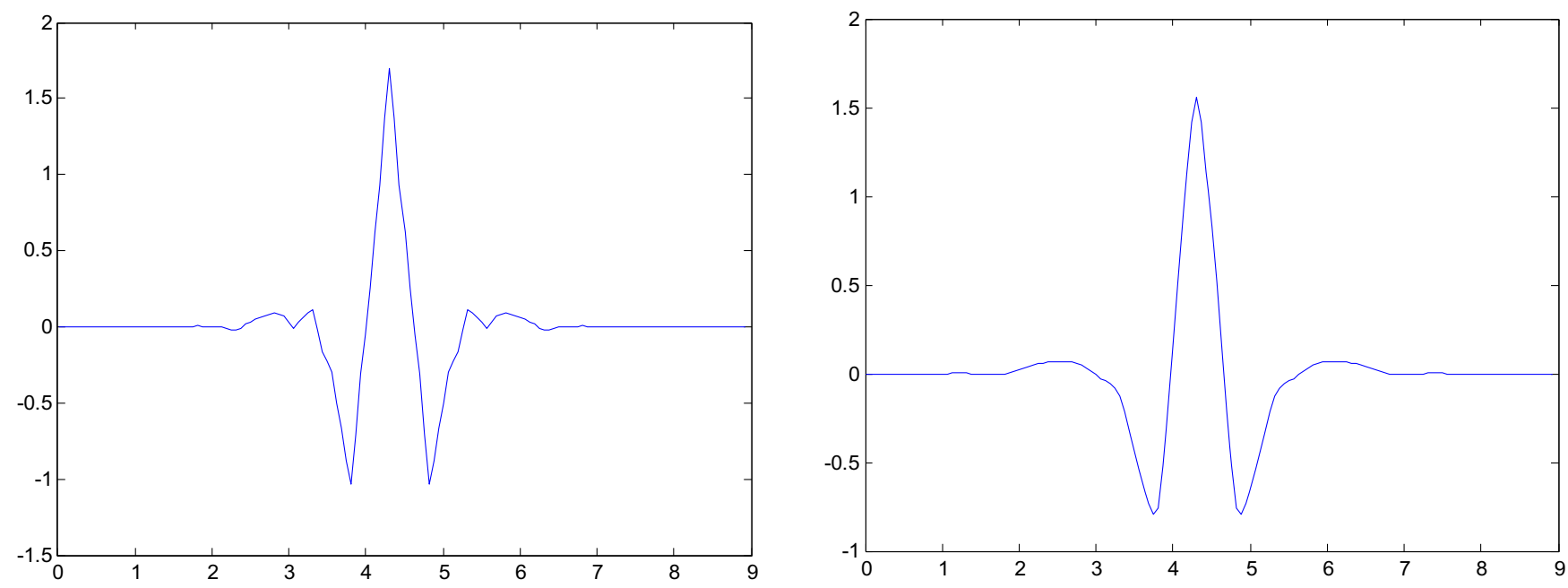

Fig. 2. rbio4.4 wavelet: decomposition (left) and reconstruction (right).
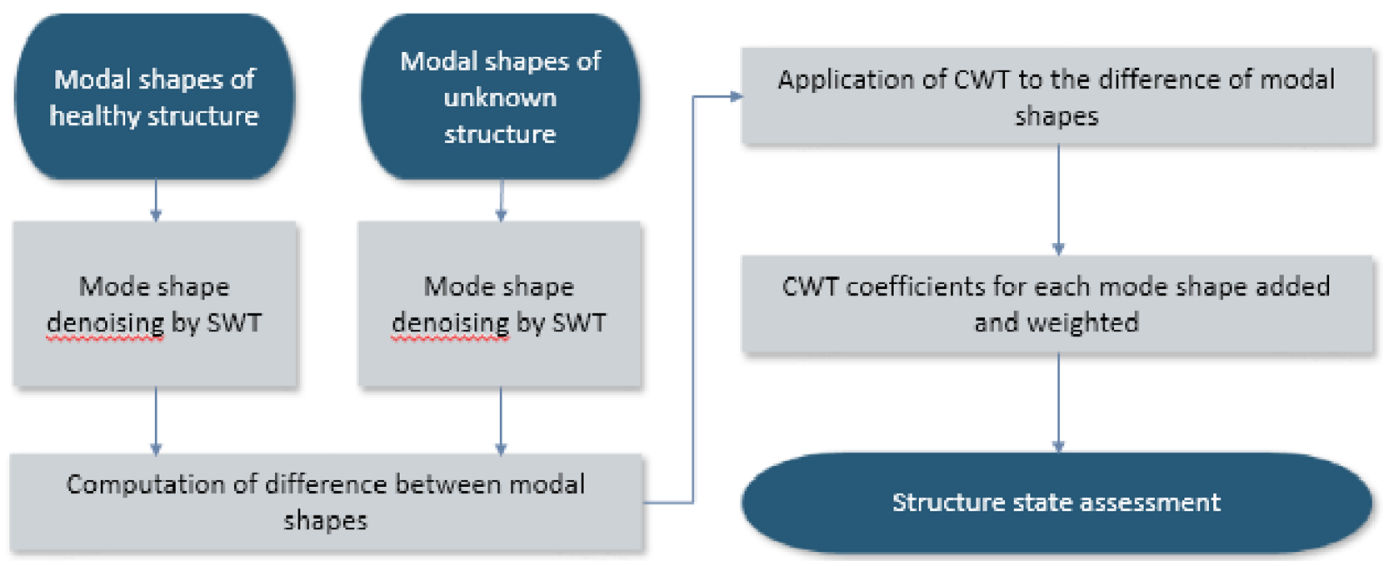

Fig. 3. Proposed methodology based on combined modal Wavelet Transform strategy.

When wavelet analysis is performed in the extended signal, the edge effect will appear at the end and beginning of the extended signal. The extended signal with boundary effects is going to be reshaped after Wavelet Transform is performed, leaving the original signal free of edge effects. In this article and after several verifications, a cubic linear extrapolation based on four neighboring points is going to be applied in both ends of the input signal. Each mode shape was also be normalized by Euclidean norm to 1.

\subsection{Refinement and difference of modal shapes}

The combination of SWT and CWT proposed by Cao and Quiao [1] consists of mode shape refinement by taking advantage of multiresolution analysis feature from SWT and then damage revealment using CWT. The refined modal shapes are the approximate coefficients resulting from analyzing the input data with SWT. If the decomposition level performed by SWT is too high, there is a risk that the discontinuity in modal shapes provoked by a damage may disappear. Moreover, it depends on the level of noise presented on the signal. In this work, a two-level decomposition with SWT will be performed to input modal shapes to refine them and obtain information related to the position of the damage.

As already stated, the methodology proposed by Cao and Quiao [1] does only applies to the first mode shape, which is considered as insufficient for damage detection since the sensitivity of a mode shape to a local damage depends on its position. Solis et al. [2] proposed a methodology to deal with this issue. They recommended a detection method based on the addition of the coefficients of the CWT of the difference of modal shapes between undamaged and damaged states of the structure. Firstly, the difference of the extended refined modal shapes is computed:

$$
\Phi_{d i f}(x)=\Phi_{d a m}(x)-\Phi_{\text {undam }}(x)
$$

After the difference of modal shapes is obtained, Wavelet Transform of the difference of modal shapes is performed. The previous step for the $i^{\text {th }}$ mode shape is written as:

$$
C W T^{i}(a, b)=\int_{-\infty}^{+\infty} \Phi_{d i f}^{i}(x) \frac{1}{\sqrt{a}} \psi^{*}\left(\frac{x-b}{a}\right) d x
$$




\subsection{Addition and weighting of mode shapes}

Zhong and Oyadiji [38] proposed a damage parameter based on the addition of the results for all modal shapes. Radziensky et al. [39] employed the change in natural frequencies for each mode to define a damage probability function which was used to weight the wavelet coefficients along the beam for each mode shape, and results for each mode shape were added up. The weighting of the coefficient is used to emphasize the most sensitive mode shapes. It is assumed that when the change in natural frequency is high, the difference between modal shapes will also be important. This way the modal shapes that do not change their natural frequencies are almost not taken into account since they do not bring new information; what is more, they could become a source of noise.

$$
C W T_{\text {weighted }}^{i}(a, b)=C W T^{i}(a, b) *\left(1-\frac{w_{u n d a m}^{i}}{w_{d a m}^{i}}\right)^{2}
$$

where $w_{\text {undam }}^{i}$ and $w_{\text {dam }}^{i}$ are natural frequencies of mode shape $i$ for undamaged and damaged states, respectively. The final step is to combine the results or each mode shape by adding them up. The result is a global wavelet coefficient.

$$
C W T_{\text {global }}(a, b)=\sum_{i=1}^{m} C W T_{\text {weighted }}^{i}(a, b)
$$

Where $m$ represent the number of modes added up.

\subsection{Normalization and analysis of the results}

The value of $C W T$ coefficients rises with the scale. This issue could leads to a misinterpretation when analyzing the coefficients for each scale in a scalogram. In fact, the values for small scales could become masked by those of higher scales. For this Wavelet Transform reason, the global Wavelet Transform coefficients are normalized to the unity for each scale to obtain clearer results:

$$
\left[C W T_{\text {norm }}\right]_{a}=\frac{\left[C W T_{\text {global }}(a, b)\right]_{a}}{\max \left[C W T_{\text {global }}(a, b)\right]_{a}}
$$

The normalized version of $C W T$ coefficients is going to be analyzed to detect and localize damage in the structure. Based on the analysis previously made, any abrupt change in the coefficients will be interpreted as the effect of a structural damage.

\section{Results}

\subsection{Presentation of the numerical simulation cases}

A 3D model built with COMSOL is going to be used to simulate the deflection of a clamped-free cracked beam (Fig. 4). The left end is fixed at the coordinates of the origin. The material properties used are: Young's modulus $E=183.236 \mathrm{MPa}$, Poisson ratio $v=0.33$ and mass density

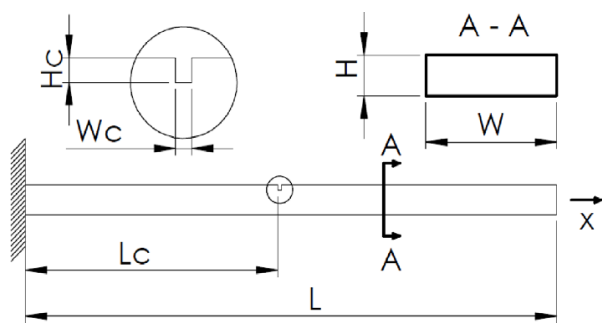

Fig. 4. Clamped-free beam model.

$\rho=7870 \mathrm{~kg} \cdot \mathrm{m}^{-3}$. Geometrical properties of the beam are length $L=1 \mathrm{~m}$, width $W=0.0258 \mathrm{~m}$ and height $H=0.0053 \mathrm{~m}$. The crack crosses the whole width of the beam. The width of the crack $W_{\mathrm{C}}$ is set to $0.1 \mathrm{~mm}$ while its height $H_{\mathrm{C}}$ is a parameter.

The finite element type employed to perform the mesh was 3D tetrahedron element provided by COMSOL software. The number of meshing points is controlled by the software and depends on the shape of the structure, thus it changes with the size of the crack. A high meshing density is applied near the crack area so that its location can be accurately assessed (Fig. 5).

The displacement of the nodes in the vertical $Y$ direction of the lower edge of the cantilever beam is selected to represent the deflection of the beam. However, only the information of 10 equally-spaced nodes was retained to simulate the position of the sensors along the beam (Fig. 6). Since damage indicators based on modal shapes require significant amount of points to accurately evaluate the state of the structure, an interpolation was performed between the values. Interpolation is also needed when measuring points are not equally spaced since they would create a non-uniform sampling input vector. In other words, damage indicators based on modal shapes must be applied to a vector with geometrically equally spaced components. If this was not the case, the measures could be interpolated to get a new vector which could fit that requirement. Another use of interpolation is to disregard measure points that can easily be detected as incorrect, whether due to noise influence or any external factor. It is also worth mentioning that there always should be measuring points at the end and the beginning of the beam.

That way, the analyzed modal shapes are the result of the interpolation of the measuring points. Extrapolation, which could add uncertainties, is never done. In this article, a cubic interpolation described in [2] was applied to obtain 128 interpolated points as shown in Figure 7.

In accordance with the previous literature review section, nine damage scenarios are chosen and simulated to analyze the behavior of damage indicators, where the position of the damage and its size are varied (Table 1 ).

\subsection{Selection of modal shapes}

It is well known that the influence of a damage becomes almost imperceptible when it lies near a node of the mode shape. The effect of damages on modal shapes is in relation with the position of the modal nodes for each mode shape In other words, not all the modal shapes have the same 


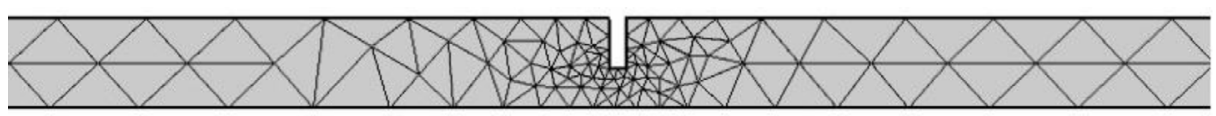

Fig. 5. Finite element mesh of the beam.

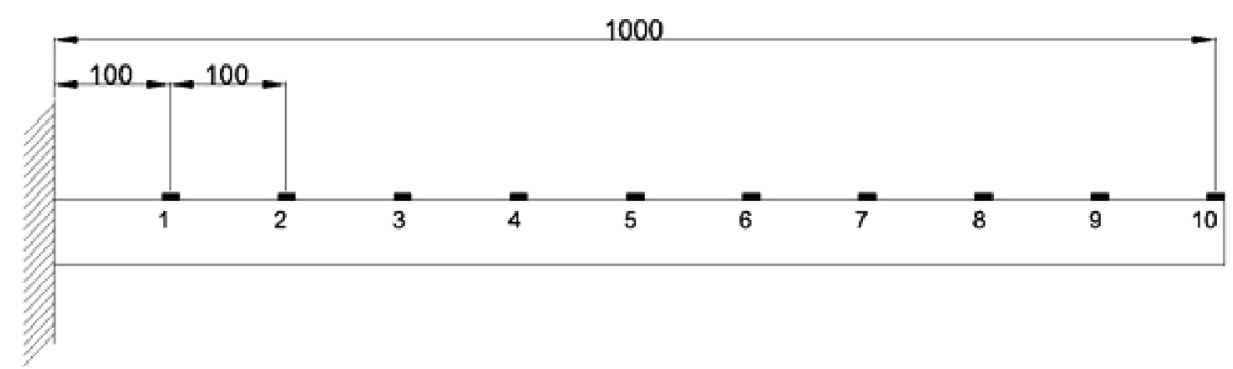

Fig. 6. Position of the accelerometers along the beam.
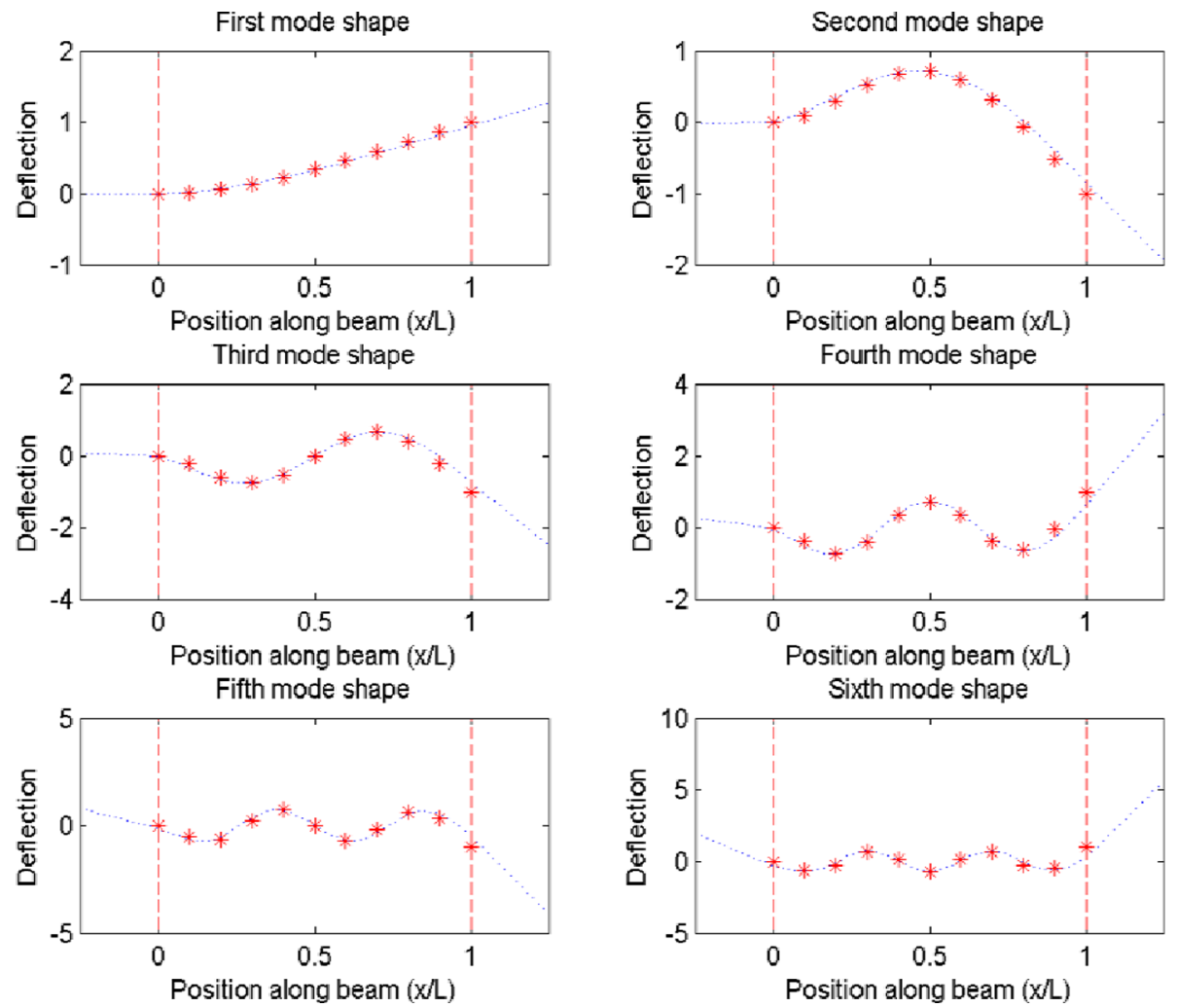

Fig. 7. The stars correspond to the measured points (10 points) while the dotted points are the interpolated modal shapes (128 points). Points beyond of the vertical lines are removed after the transform is performed.

Table 1. Damage cases studied.

\begin{tabular}{llllllllll}
\hline Damage case & 1 & 2 & 3 & 4 & 5 & 6 & 7 & 8 & 9 \\
\hline Location $\left(\boldsymbol{L}_{\mathbf{c}}\right)[\mathbf{m m}]$ & 100 & 100 & 100 & 500 & 500 & 500 & 900 & 900 & 900 \\
Severity $\left(\boldsymbol{H}_{\boldsymbol{c}} / \boldsymbol{H}\right)[\boldsymbol{\%}]$ & 1 & 10 & 25 & 1 & 10 & 25 & 1 & 10 & 25 \\
\hline
\end{tabular}


Table 2. Frequencies and MAC coefficient for each modal shape for the damage scenario Case $5\left(L_{\mathrm{c}}=500 \mathrm{~mm}, H_{c}\right)$ $H=10 \%$ ). Identification of the most sensitive mode shape weighting of modal shapes in order to reduce those unwanted effects.

\begin{tabular}{lllllll}
\hline Mode & 1 & 2 & 3 & 4 & 5 & 6 \\
\hline Undamaged frequencies [Hz] & 4.1311 & 25.8896 & 72.4918 & 142.055 & 234.827 & 350.791 \\
Damaged frequencies [Hz] & 4.12355 & 25.6877 & 72.4916 & 140.976 & 234.819 & 348.17 \\
Relative difference of frequencies [\%] & 0.1846 & 0.77981 & 0.00025 & 0.75897 & 0.00320 & 0.746 \\
MAC coefficient & 0.99999 & 0.99993 & 0.99999 & 0.99958 & 0.99999 & 0.99881 \\
\hline
\end{tabular}
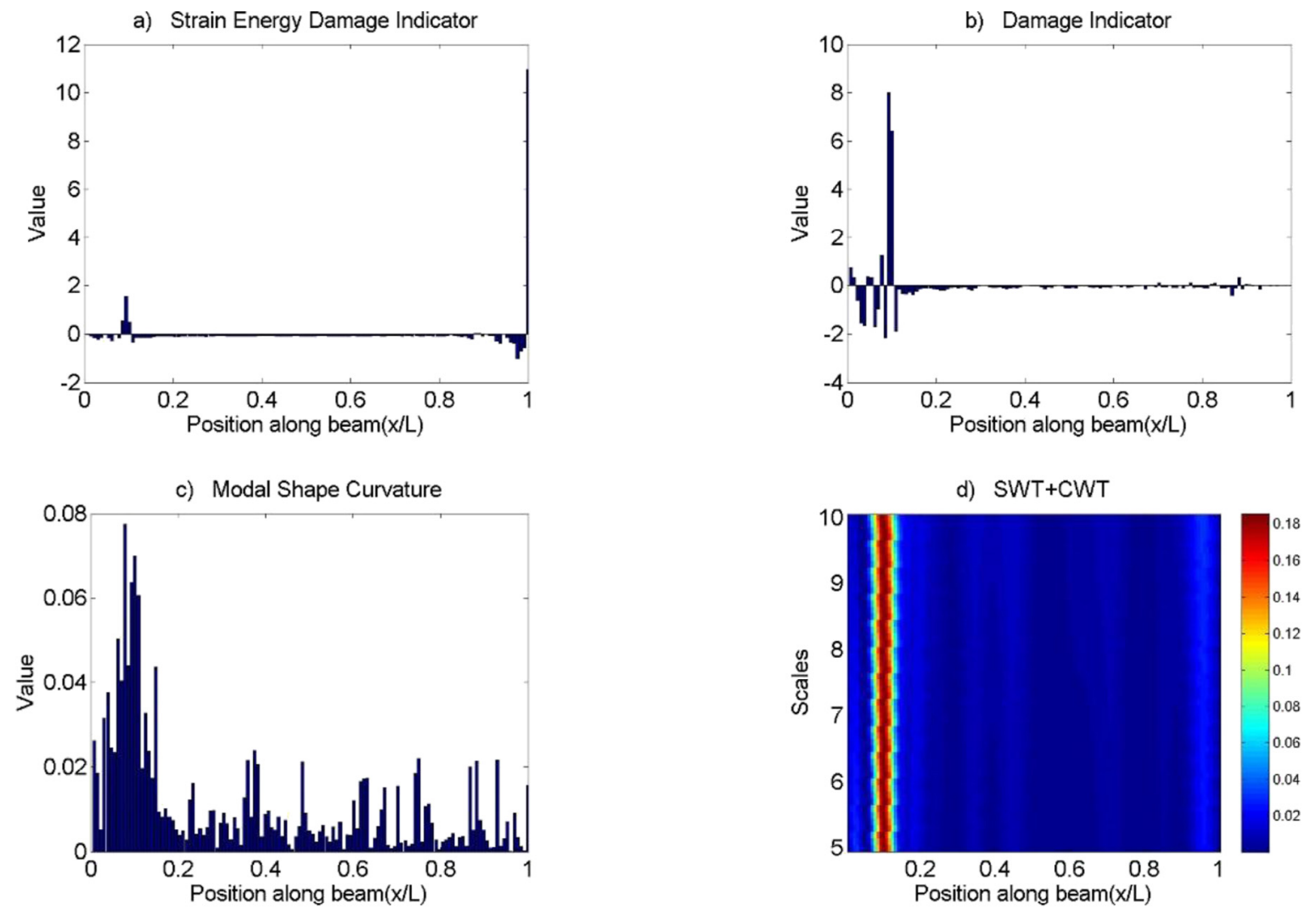

Fig. 8. Comparison of damage indicators for the Case 1: a) Strain Energy Damage Indicator, b) Damage Index Indicator, c) Modal Shape Curvature Indicator, d) SWT + CWT Wavelet Analysis.

sensitiveness to a particular damage. Furthermore, more important changes in natural frequencies are expected for the most sensitive modal shapes. To illustrate this effect, changes in natural frequencies and in Modal Assurance Criterion (MAC) in Table 2 are shown for Case 5. Concerning the changes in natural frequencies, it is possible to conclude that modes 1, 2, 4 and 6 are the most sensitive to the particular damage scenario. On the other hand, modes 3 and 5 almost does not show any change but it's completely normal due to the damage position and node of vibration of these modes. This conclusion can be reinforced by analyzing MAC coefficients since they show the correlation between the analyzed mode shape and its undamaged version (value of 1 ). In other words, damage detection and localization cannot rely in only one single mode shape. This scenario justifies the weighting of modal shapes in order to reduce those unwanted effects.
On the other hand, mode 6 may be able to offer useful information, however ten sensors are not considered enough to correctly interpolate it. Therefore, for the rest of the paper there are analyzed the first 4 modes, since from a practical point of view, the lowest modal shapes are the most suitable to obtain.

\subsection{Effects of crack location and size}

The proposed methodology based on Wavelet Transform will be compared with some of the most known damage indicators in the bibliography: Strain Energy Damage Indicator (SEDI), Damage Index Indicator (DI) and Modal Shape Curvature (MSC).

Figures 8-16 show the behavior of damage indicators for all damage cases, which are described in Table 1. Figures 7-9 confirm the hypotesis already made that the 
a) Strain Energy Damage Indicator

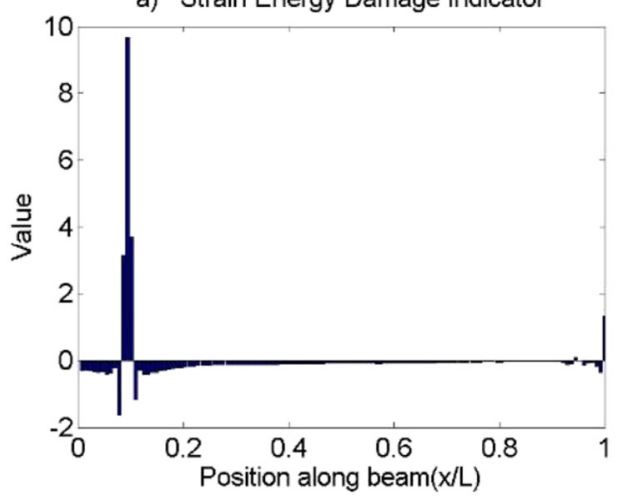

c) Modal Shape Curvature

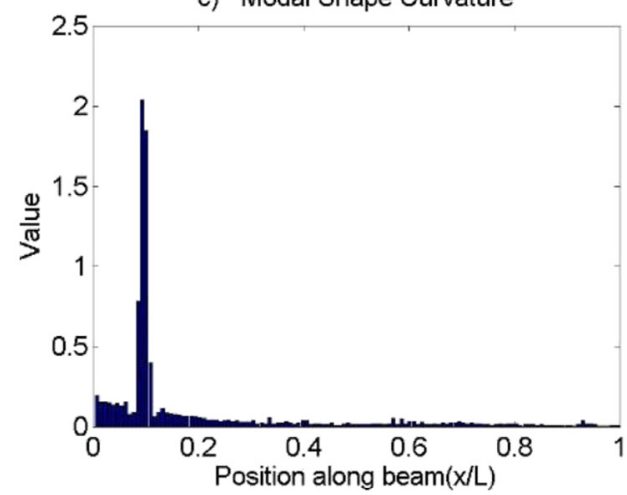

b) Damage Indicator

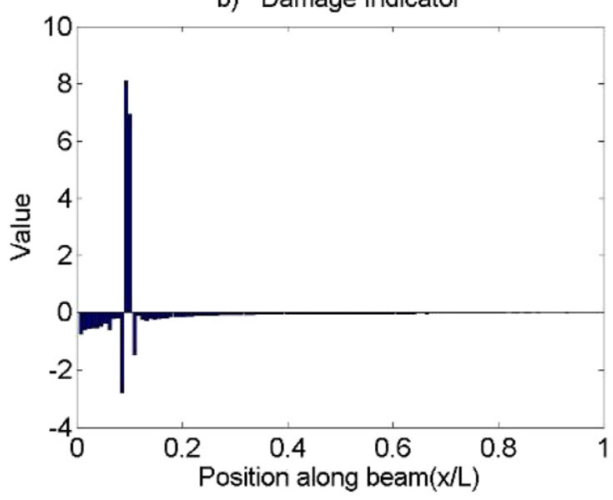

d) SWT+CWT

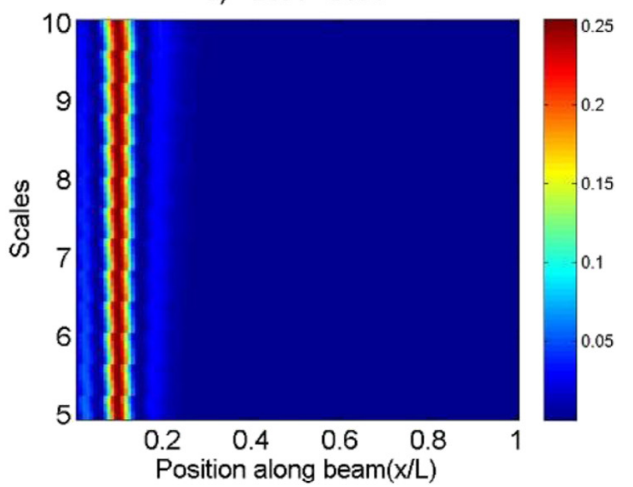

Fig. 9. Comparison of damage indicators for the Case 2: a) Strain Energy Damage Indicator, b) Damage Index Indicator, c) Modal Shape Curvature Indicator, d) SWT + CWT Wavelet Analysis.

beginning of the beam is a problematic zone to detect and localize damage. In Figure 7, it can be objected that SEDI completely fails to locate the damage when it is small and close to the beginning of the beam. On the other hand, the maximum absolute coefficients of DI and MSC do not coincide with the actual position of fissure. However the influence of computational noise is remarkable; on a practical situation, a smaller damage may be hidden in those situations. Figures 8 and 9 show that these effects are reduced when the damage size is increased since the difference between the high values produced by the damage and those provoked by noise increases. In the case of Wavelet Analysis, damage's location is correctly assessed and does not give doubts about the actual position of the damage.

Figures $10-12$ show the behavior of damage indicator when the damage is in the middle of the beam. The maximum absolute coefficients of SEDI, DI and MSC do coincide with the actual position of the damage for the three cases; the estimation on the location of the damage is exact. On the other hand, the location of these damage indicators is not precise since uncertain zones are presented near the maximum coefficients. In Figure 10 the peaks on both sides of the maximum coefficients may be easily confounded with two independent damages. This effect is attenuated when the size of the damage increases. In the case of Wavelet Analysis, this problem is partly solved by weighting the modal shapes that may introduce noise to the final result.
Figures 13-15 illustrate that damages in the end zone of the beam are the most easily located. All indicators can detect and correctly assess the location of the damage. Moreover, the computational noise is less compared to the one in the beginning and middle cases. Wavelet Analysis has also the most solid performance in these cases since the computational noise is almost imperceptible.

\subsection{Accuracy of damage location}

To compare the accuracy of damage location, the position of the maximum absolute coefficients for each damage indicator is considered as its estimation to damage's position (Table 3). In the case of CWT, a multiscale analysis is performed which gives different coefficients for each scale. By equation (2) it can be deduced that big scales are prone to general characteristics of the signal while small scales are more sensitive to local events. Since damages provoke a local discontinuity in modal shapes, scale 5 is chosen in this paper to evaluate the location accuracy of wavelet methodology. For the nine cases illustrated in the Figures 7-15, it can be concluded that the damage localization accuracy of the analyzed damage indicators does not depend on the size of the damage. The location accuracy of damage indicators should be analyzed with respect to the spatial distance between the simulated sensors. In the proposed case, the corresponded distance is $0.1 \mathrm{~m}$, therefore a threshold of $0.1 \mathrm{~m}$ to both sides of the certain location of the damage can be defined. 
a) Strain Energy Damage Indicator

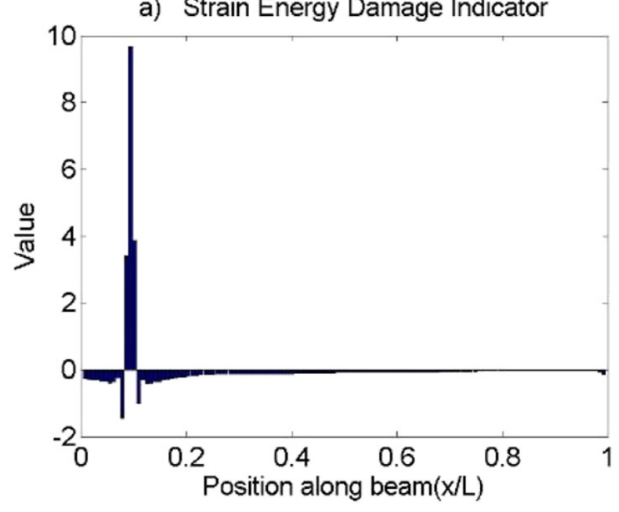

c) Modal Shape Curvature

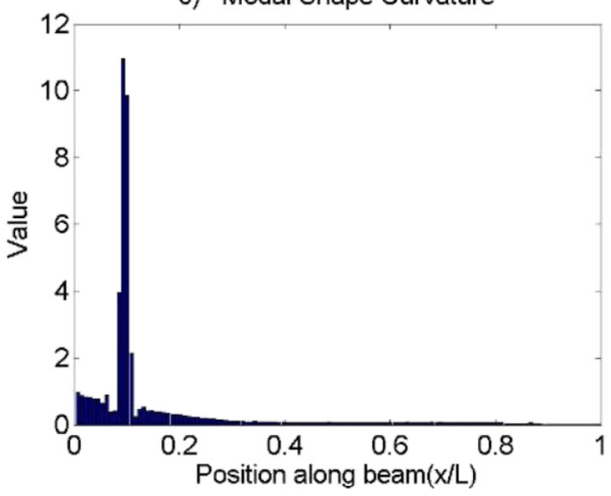

b) Damage Indicator

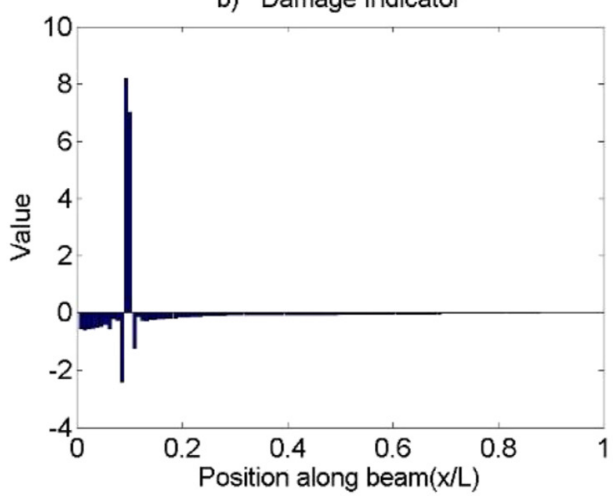

d) SWT+CWT

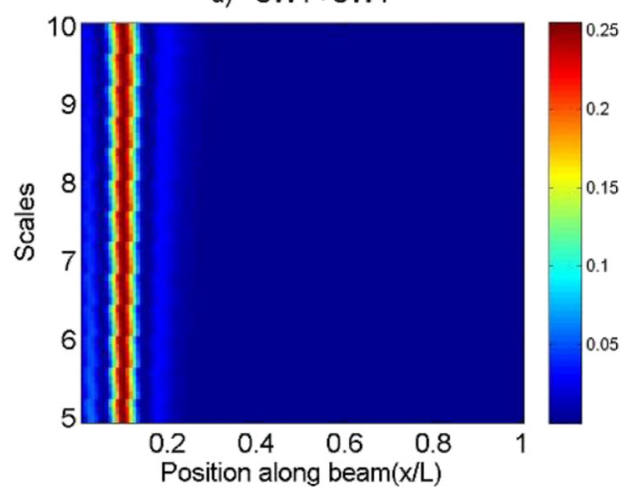

Fig. 10. Comparison of damage indicators for Case 3: a) Strain Energy Damage Indicator, b) Damage Index Indicator, c) Modal Shape Curvature Indicator, d) SWT + CWT Wavelet Analysis.

a) Strain Energy Damage Indicator
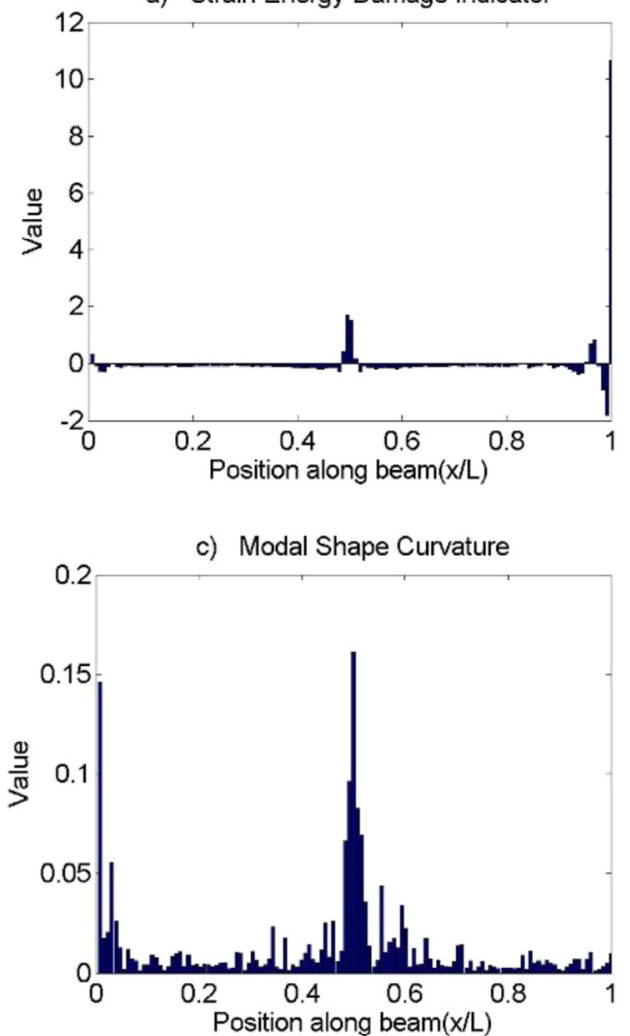

b) Damage Indicator

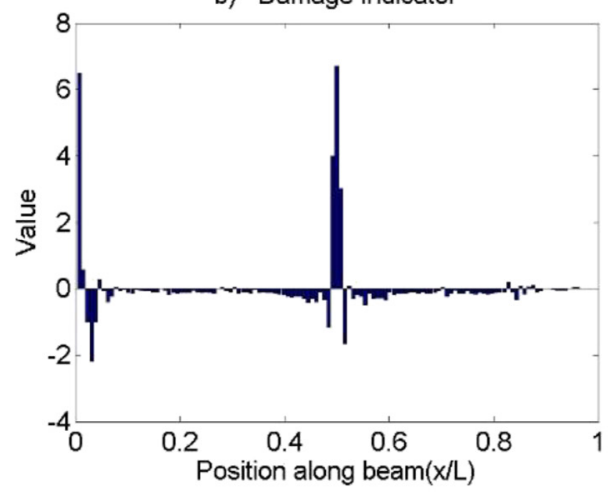

d) $\mathrm{SWT}+\mathrm{CWT}$

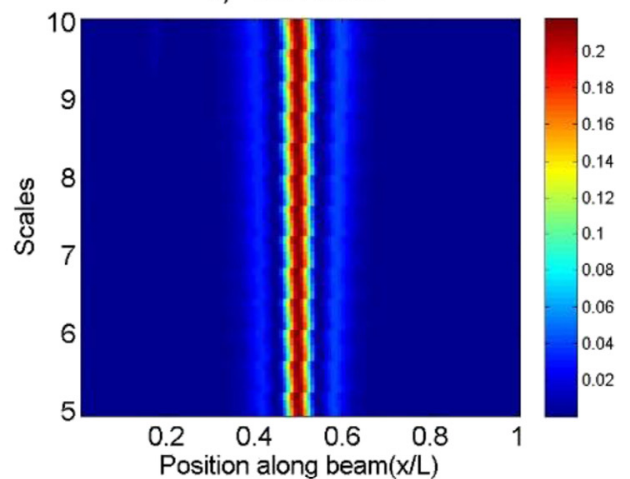

Fig. 11. Comparison of damage indicators for Case 4: a) Strain Energy Damage Indicator, b) Damage Index Indicator, c) Modal Shape Curvature Indicator, d) SWT + CWT Wavelet Analysis. 
a) Strain Energy Damage Indicator

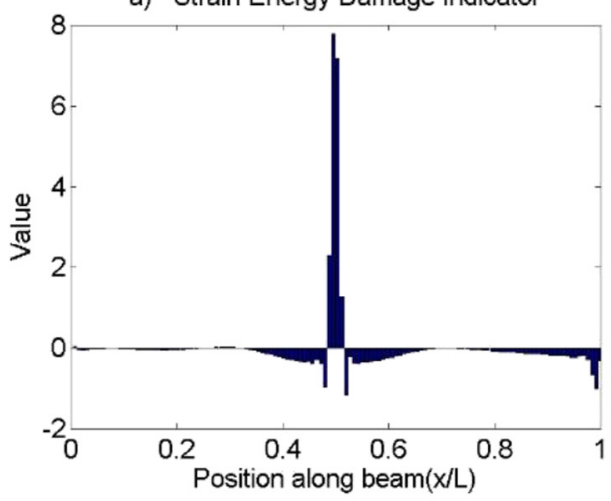

c) Modal Shape Curvature

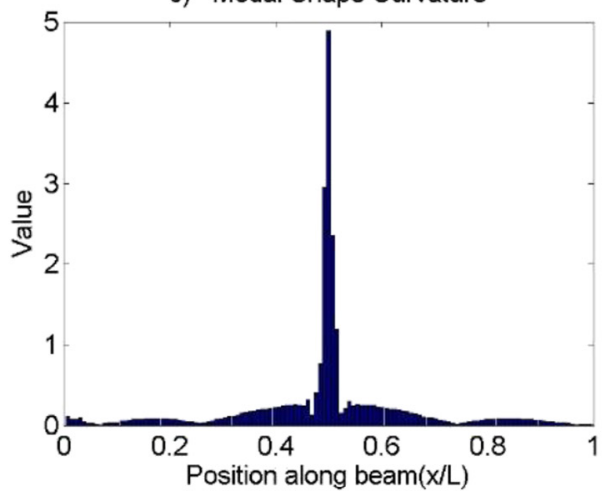

b) Damage Indicator

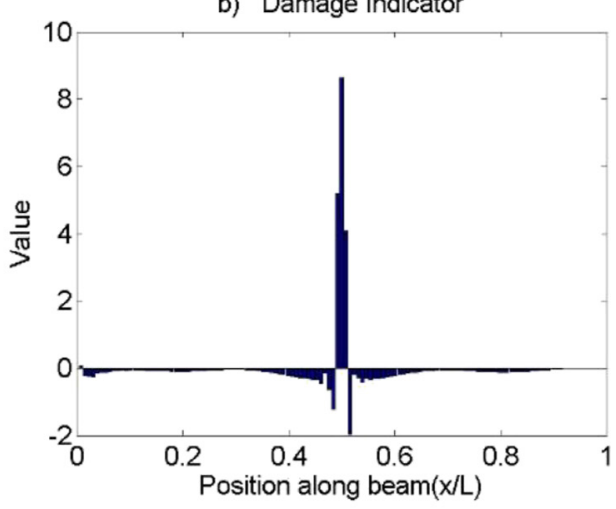

d) $\mathrm{SWT}+\mathrm{CWT}$

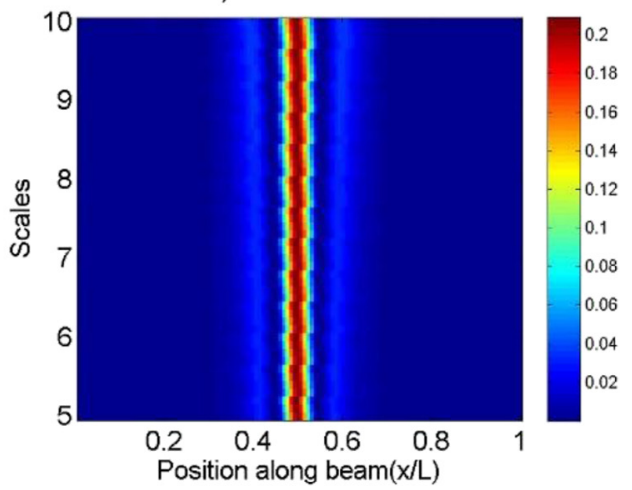

Fig. 12. Comparison of damage indicators for Case 5: a) Strain Energy Damage Indicator, b) Damage Index Indicator, c) Modal Shape Curvature Indicator, d) SWT + CWT Wavelet Analysis.

a) Strain Energy Damage Indicator

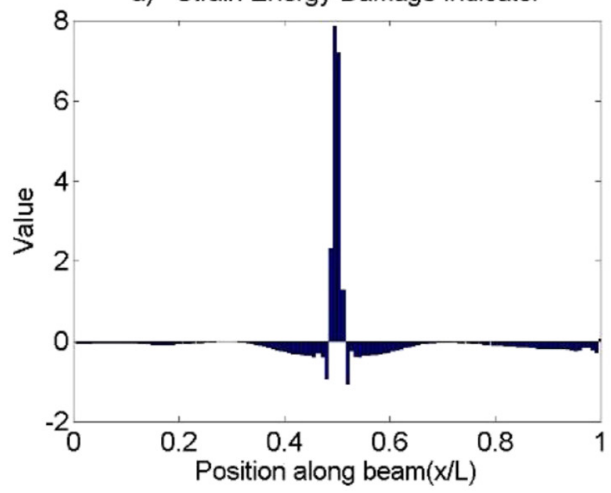

c) Modal Shape Curvature

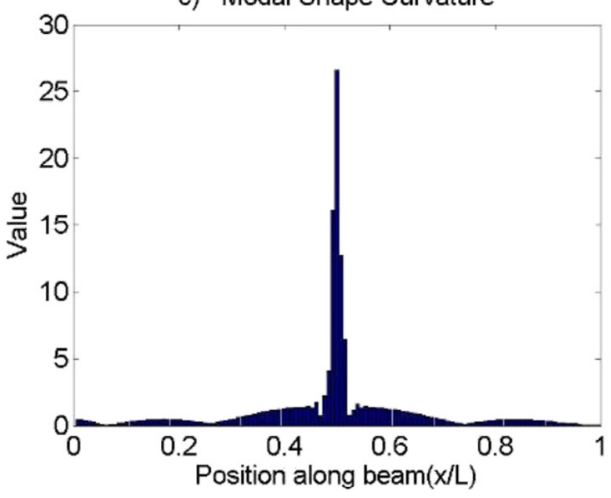

b) Damage Indicator

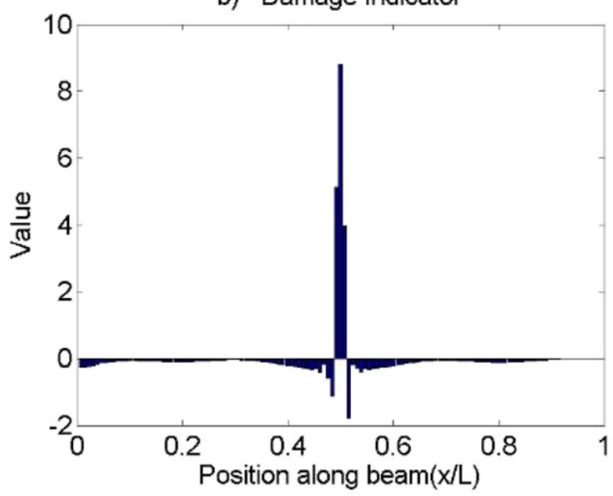

d) $\mathrm{SWT}+\mathrm{CWT}$

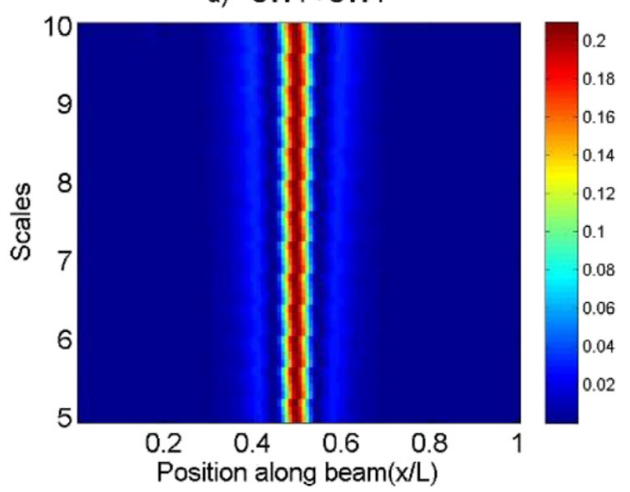

Fig. 13. Comparison of damage indicators for Case 6: a) Strain Energy Damage Indicator, b) Damage Index Indicator, c) Modal Shape Curvature Indicator, d) SWT + CWT Wavelet Analysis. 
a) Strain Energy Damage Indicator
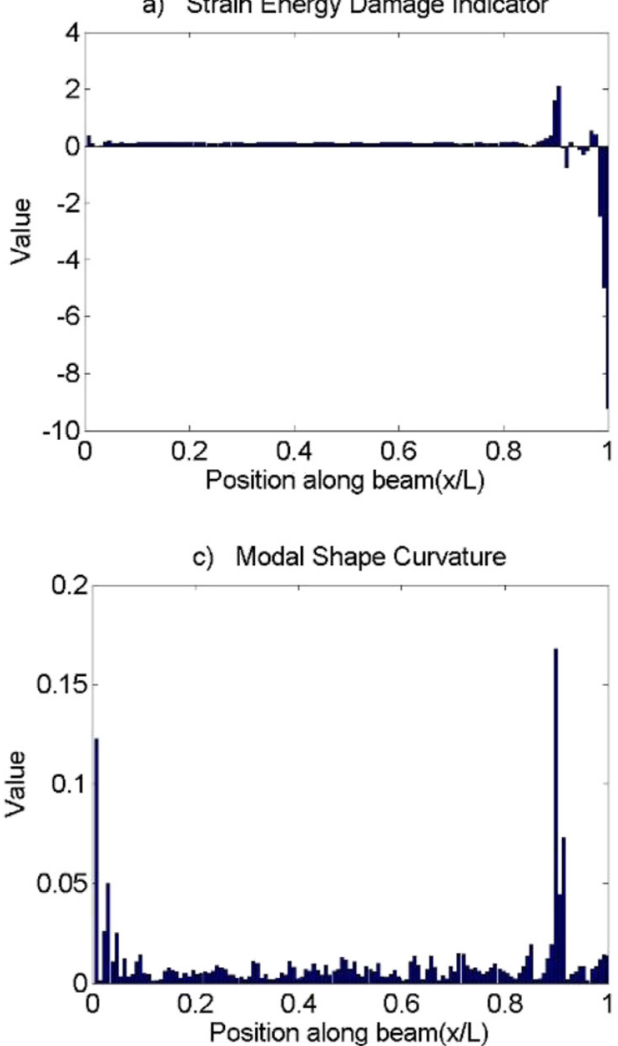

b) Damage Indicator

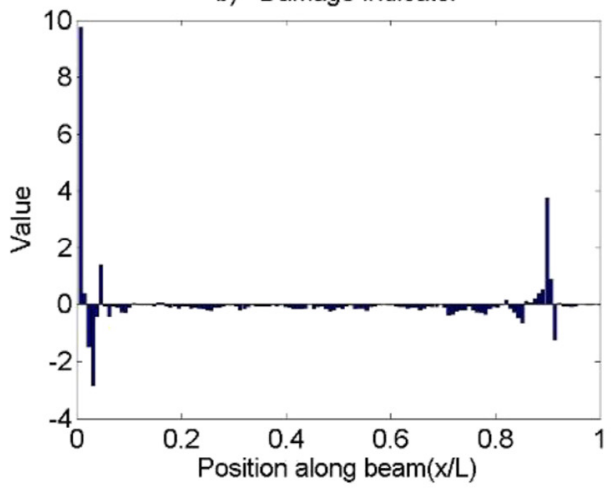

d) SWT+CWT

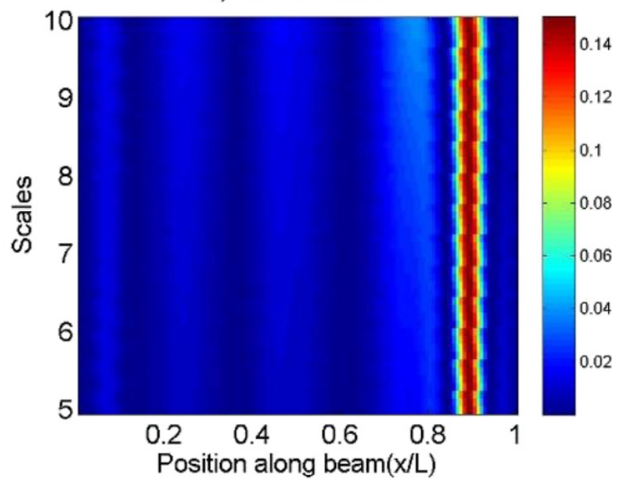

Fig. 14. Comparison of damage indicators for Case 7: a) Strain Energy Damage Indicator, b) Damage Index Indicator, c) Modal Shape Curvature Indicator, d) SWT + CWT Wavelet Analysis.

a) Strain Energy Damage Indicator

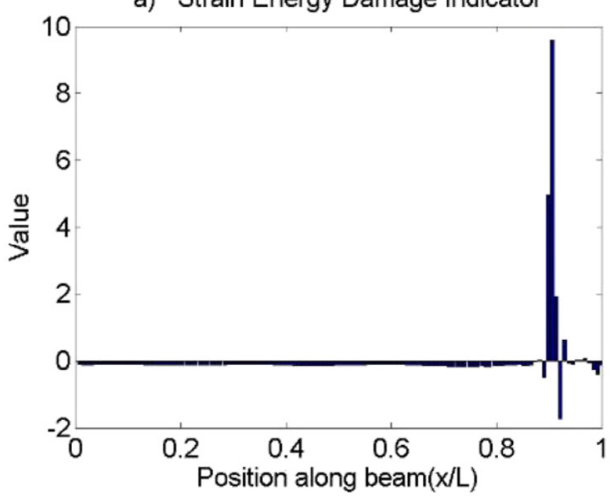

c) Modal Shape Curvature

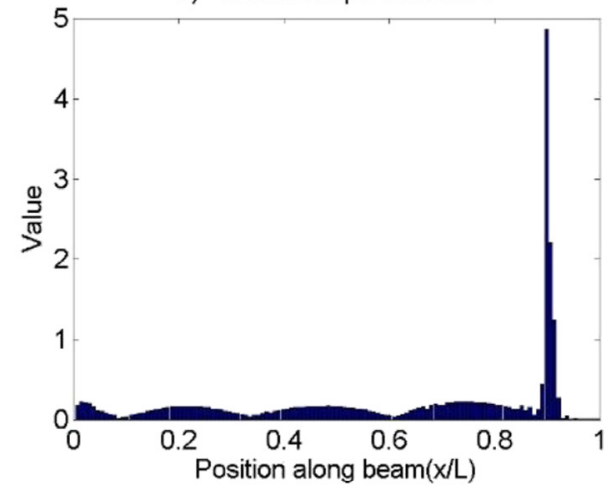

b) Damage Indicator

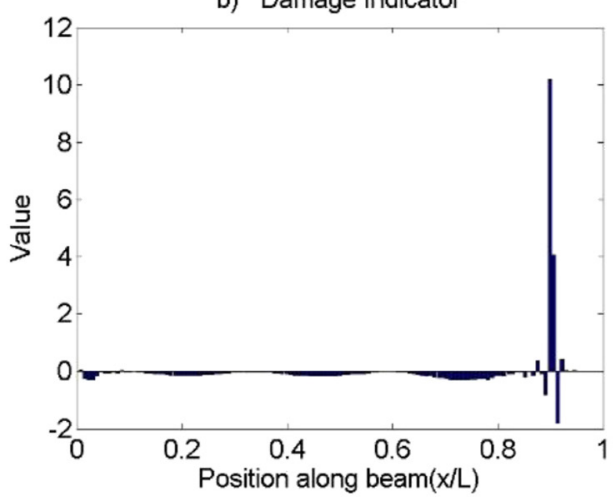

d) SWT+CWT

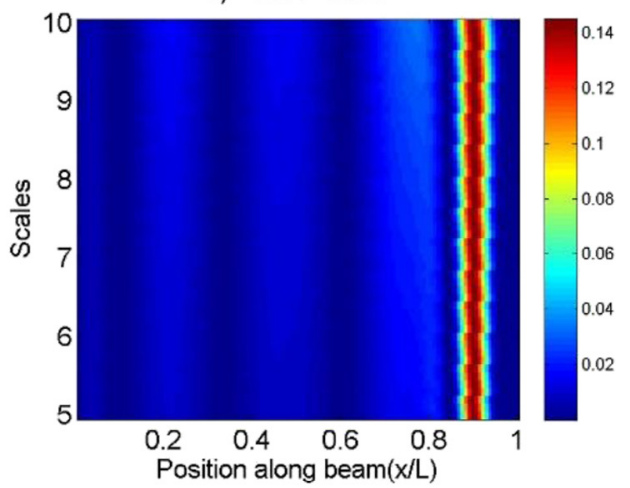

Fig. 15. Comparison of damage indicators for Case 8: a) Strain Energy Damage Indicator, b) Damage Index Indicator, c) Modal Shape Curvature Indicator, d) SWT + CWT Wavelet Analysis. 
Table 3. Position of maximum coefficient for each damage indicator vs position of the fissure.

\begin{tabular}{lllll}
\hline Position of the damage & SEDI & DI & MSC & Wavelet \\
\hline$L_{\mathrm{c}}=0.1 \mathrm{~m}$ & 0.0945 & 0.0938 & 0.0938 & 0.1016 \\
$L_{\mathrm{c}}=0.5 \mathrm{~m}$ & 0.4961 & 0.5000 & 0.5000 & 0.5000 \\
$L_{\mathrm{c}}=0.9 \mathrm{~m}$ & 0.9055 & 0.8984 & 0.8984 & 0.8984 \\
\hline
\end{tabular}

Table 4. Relative position of maximum coefficient for each damage indicator vs position of the fissure, regarding the spatial distance between simulated sensors $(0.1 \mathrm{~m})$.

\begin{tabular}{lllll}
\hline Position of the damage & SEDI & DI & MSC & Wavelet \\
\hline$L_{\mathrm{c}}=0.1 \mathrm{~m}$ & 0.0551 & 0.0625 & 0.0625 & 0.0156 \\
$L_{\mathrm{c}}=0.1 \mathrm{~m}$ & 0.0394 & 0 & 0 & 0 \\
$L_{\mathrm{c}}=0.1 \mathrm{~m}$ & 0.0551 & 0.0156 & 0.0156 & 0.0156 \\
\hline
\end{tabular}

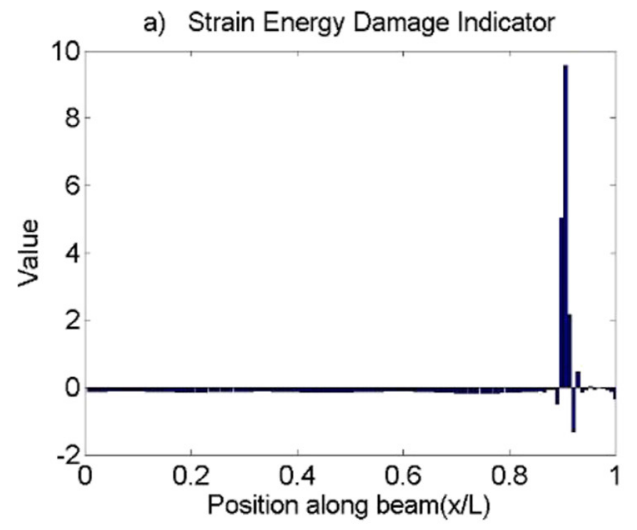

c) Modal Shape Curvature

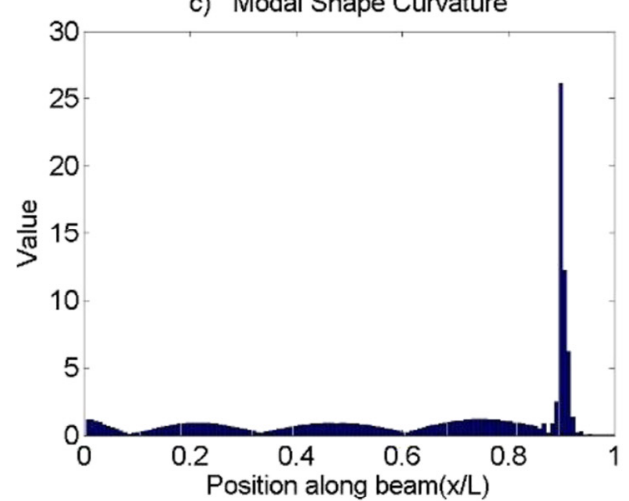

b) Damage Indicator

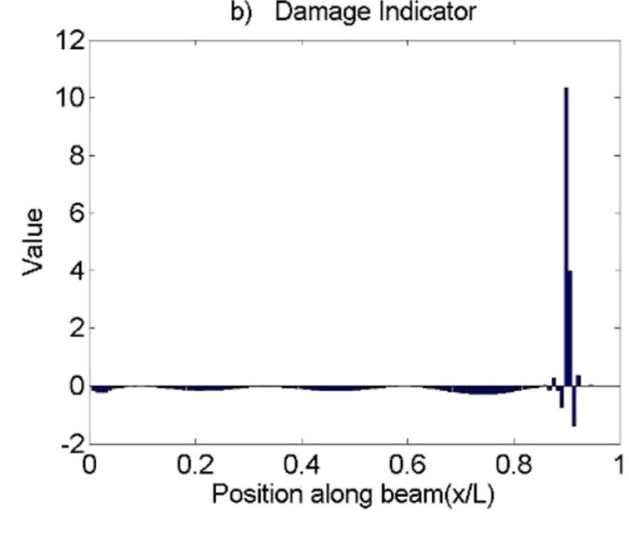

d) SWT+CWT

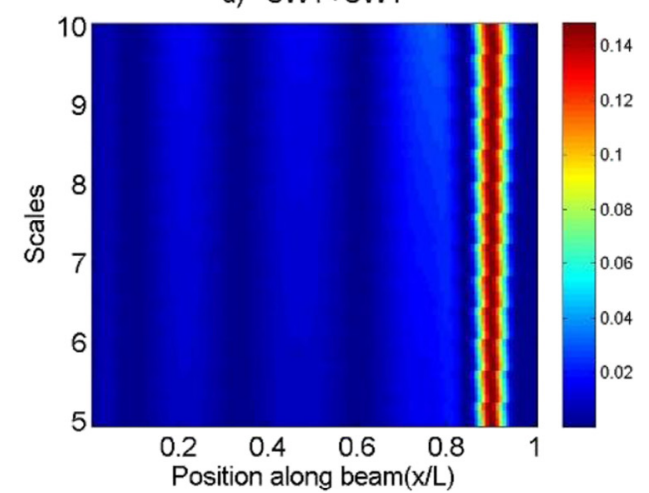

Fig. 16. Comparison of damage indicators for Case 9: a) Strain Energy Damage Indicator, b) Damage Index Indicator, c) Modal Shape Curvature Indicator, d) SWT + CWT Wavelet Analysis.

The spatial distance between sensors can be taken into account with the following formula:

$$
L_{\text {relative }}=\left|\frac{L_{\text {result }}-L_{\mathrm{c}}}{D_{\text {sensors }}}\right|
$$

where $D_{\text {sensors }}$ is the spatial distance between simulated sensors, $L_{\mathrm{c}}$ is the real positon of the damage and $L_{\text {result }}$ is the damage location estimated by damage indicators.
If the indicator result is within the threshold zone, the estimation of damage position can be assessed as correct. In terms of Table 4, a good assessment of damage location is indicated with coefficients lesser than 0.1. When the damage was located close to the beginning of the beam, only Wavelet Analysis and MSC indicators were able to assess its position with accuracy. In the middle case, all the indicators proved to have the same level of performance. In the last case, Wavelet Analysis and SEDI gave good enough results while the 
a) Strain Energy Damage Indicator

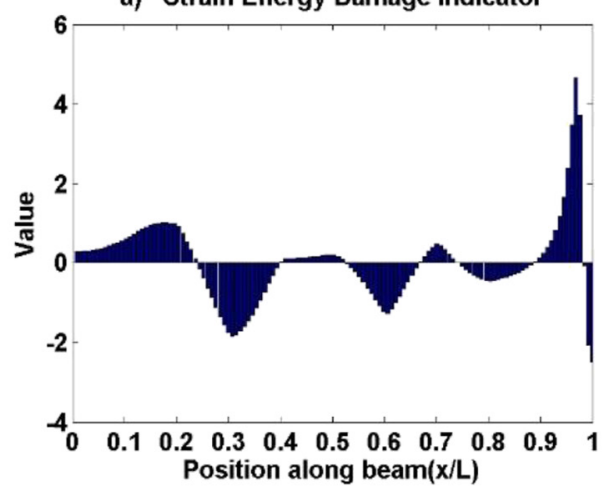

c) Modal Shape Curvature

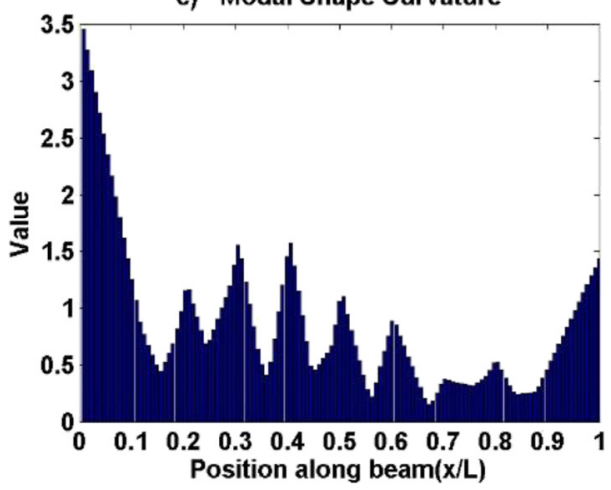

b) Damage Indicator

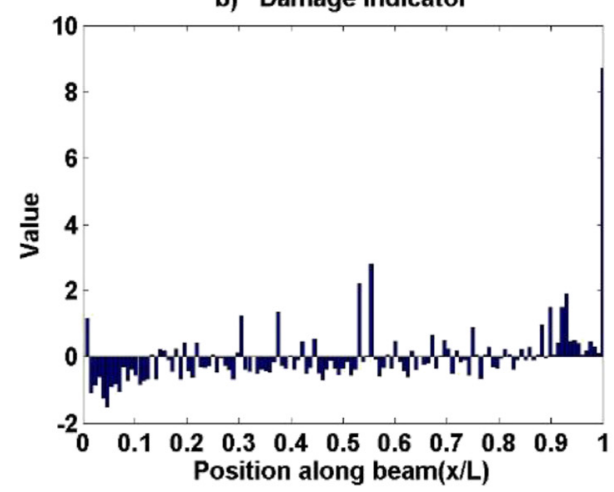

d) SWT+CWT

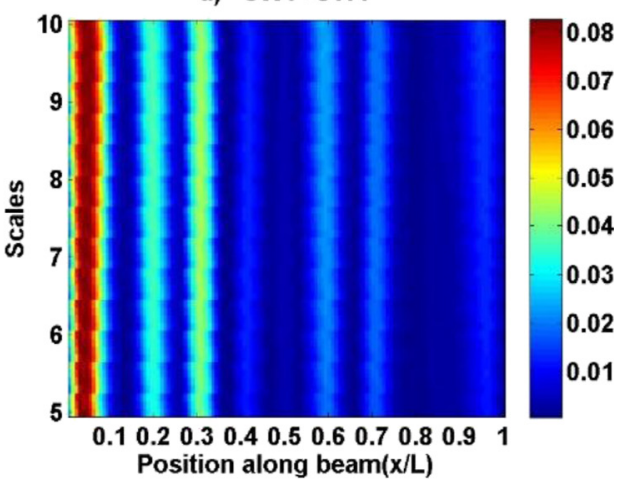

Fig. 17. Comparison of damage indicators for Case 3 with noisy signals: a) Strain Energy Damage Indicator, b) Damage Index Indicator, c) Modal Shape Curvature Indicator, d) SWT + CWT Wavelet Analysis.

a) Strain Energy Damage Indicator

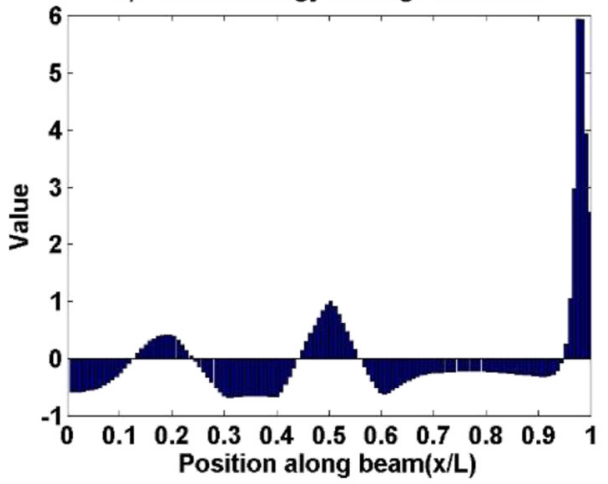

c) Modal Shape Curvature

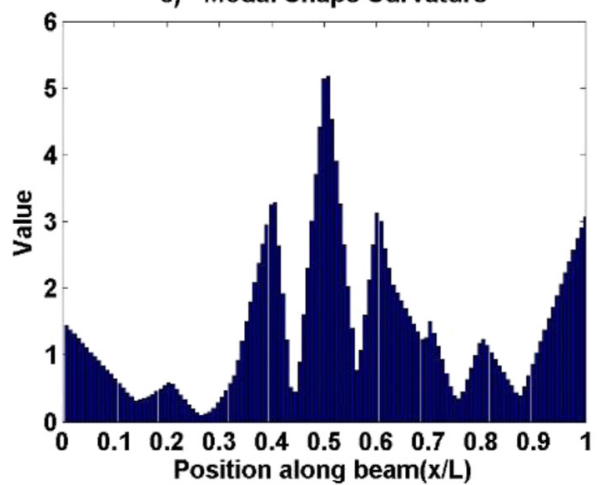

b) Damage Indicator

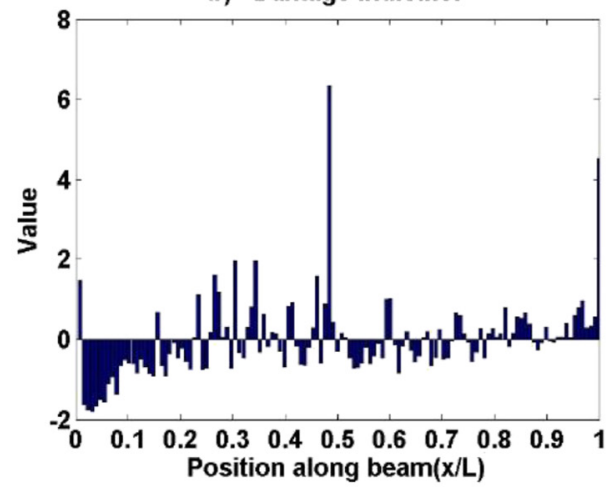

d) SWT+CWT

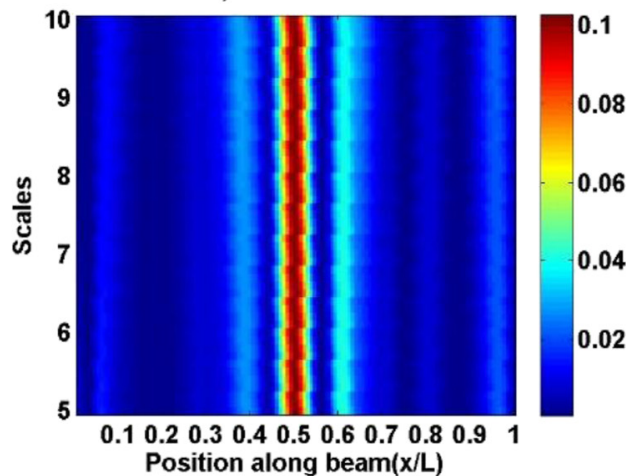

Fig. 18. Comparison of damage indicators for Case 6 with noisy signals: a) Strain Energy Damage Indicator, b) Damage Index Indicator, c) Modal Shape Curvature Indicator, d) SWT + CWT Wavelet Analysis. 
a) Strain Energy Damage Indicator

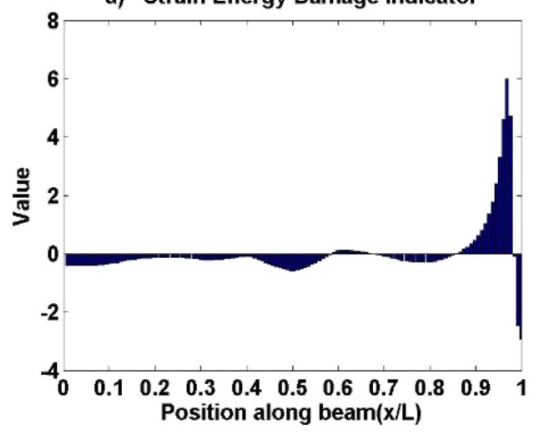

c) Modal Shape Curvature

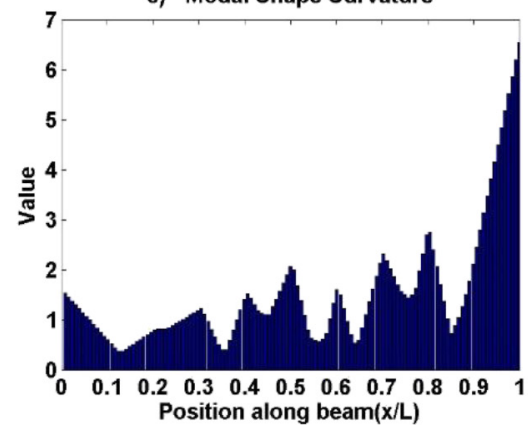

b) Damage Indicator

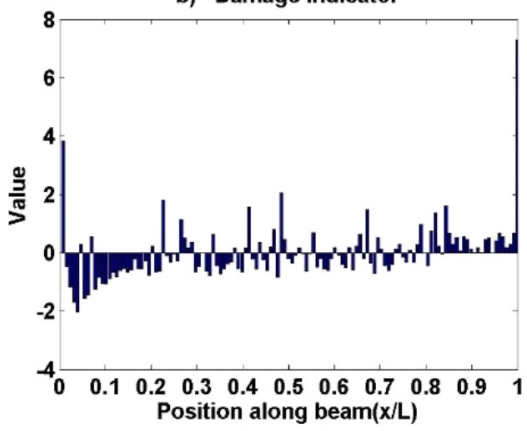

d) SWT+CWT

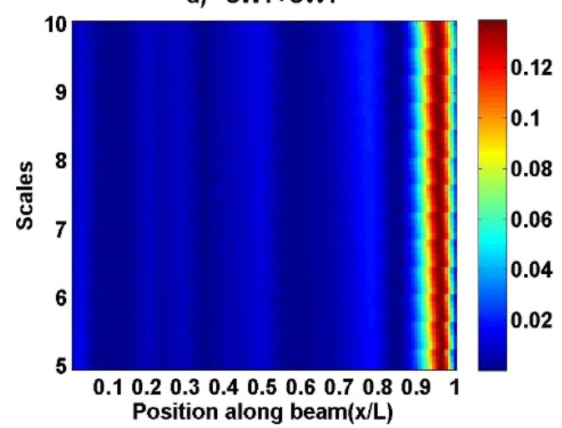

Fig. 19. Comparison of damage indicators for Case 9 with noisy signals: a) Strain Energy Damage Indicator, b) Damage Index Indicator, c) Modal Shape Curvature Indicator, d) SWT + CWT Wavelet Analysis.

assessment of DI was almost perfect. On the other hand, MSC fails in this case. Overall, it can be concluded that the methodology based on Wavelet Analysis is the most trustworthy in terms of accuracy of damage detection since in all the cases the assessed position of the damage position was close enough to the real position of it.

\subsection{Influence of noise}

In order to evaluate the detectability of damage indicators in real measurement conditions, normal distributed white noise was added to the modal shapes. The measurement of noise intensity added, defined as the power of the desired signal to the noise power, was $\mathrm{SNR}=65 \%$.

Crack localization is easier in case of large damage since if it was small, it can be easily confounded with noise. In fact, Figures 17-19 show the behavior of damage indicators to noisy signals for Cases 3, 6 and 9 for small damage ratio. Clearly Wavelet Analysis outperforms classical indicators in terms of noisy signals, giving accurate and precise assessment of damage location in all the cases. On the other hand, classical indicators were not able to cope clearly with the challenge.

A multiscale analysis using CWT can also be performed to identify the damage position in a noisy signal. It is well known that wavelet coefficients increase with the scale in presence of a discontinuity. Figure 20 is a 3D plot of the wavelet coefficients without normalization of Case 6 . Local maximum can be detected in different positions along the beam. However, those coefficients do not decrease with scale smoothly; on the other hand, they tend to be constant. This behavior can be used as a tool to differentiate the effect of the damage to the effect of the noise by looking at the decay behavior of wavelet coefficients. It can be seen that at $x=0.5 \mathrm{~m}$ the decay behavior is more important than in the rest of the beam, which indicates the presence of a damage.

\section{Conclusions}

A methodology for identifying damage in structures was proposed. The fundamental modal shapes of a potentially damaged structure are refined with SWT multiresolution analysis to separate noise and intererences components from damage-related information. The differences between the refined modal shapes and those corresponding to the safe state of the structure are afterwards computed. CWT is applied, due to its ability to perform multiscale analysis, to the difference of mode shapes. The resulting coefficients are weighted depending on the changes in natural frequencies to reduce the potential sources of noise. Finally, the coefficients are added up and normalized to obtain a single result which enables fast detection and location of the damage. A comparison between the proposed methodology and well-known classical indicators was carried out. The results proved that the proposed algorithm gives better results than classical indicators with regard to accurate location of damage. It also showed indiference to the size of damage, showing sensitivity to little damages. Furthermore, the proposed methodology outperforms the previous indicators capacity of detecting and locating damages in the presence of noise. In the futur, 


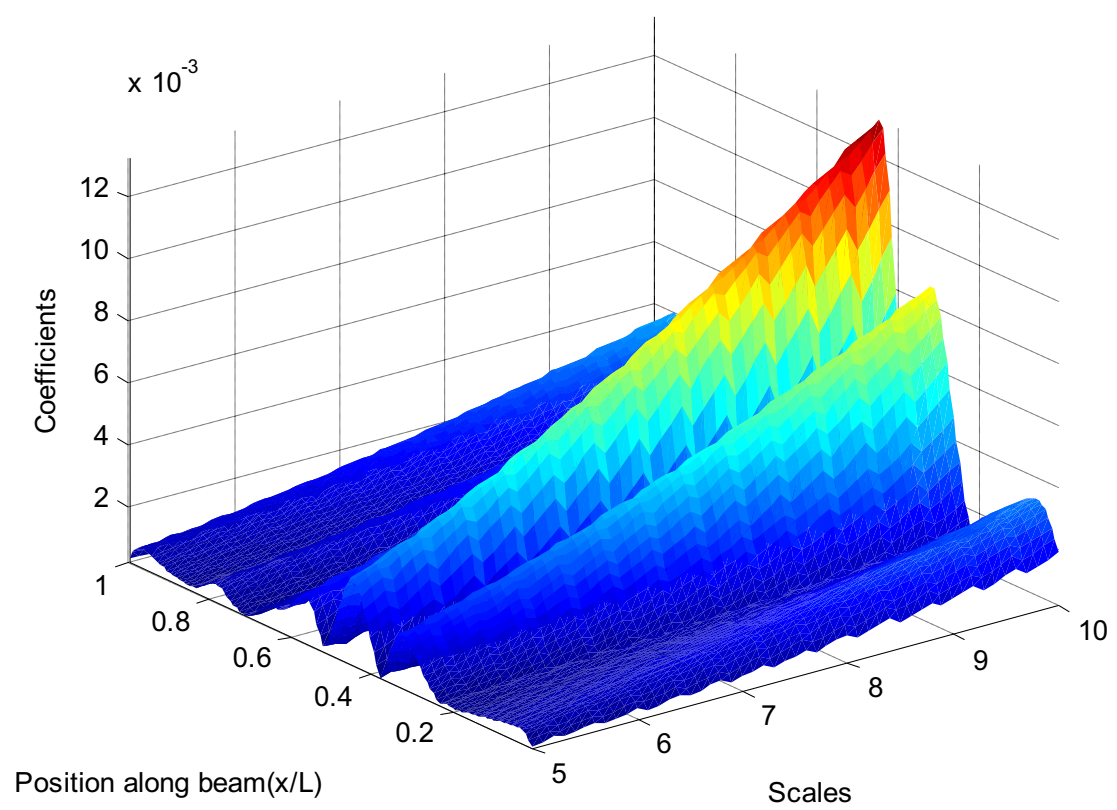

Fig. 20. Three-dimensional plot of wavelet coefficients for noisy data, Case 5 .

the methodology will be tested with experimental data in order to validate its efficiency in real cases.

\section{References}

[1] M. Cao, Q. Pizhong, Integrated Wavelet Transform and its application to vibration mode shapes for the damage detection of beam-type structures., Smart Mater. Struc. 17 (2008) 055014, DOI: 10.1088/0964-1726/17/5/055014

[2] M. Solis, M. Algaba, P. Galvan, A combined wavelet-modal analysis for damage location in beams Proc., Int. Conf. Noise Vib. Eng. ISMA 2012 (2013) 777-790, http://past.ismaisaac. be/downloads/isma2012/papers/isma2012_0780.pdf

[3] P. Cawley, R.D. Adams, The location of defects in structures from measurements of natural frequencies, J. Strain Anal. Eng. Des. 14 (1979) 49-57, DOI: 10.1243/03093247V142049

[4] O.S. Salawu, Detection of structural damage through changes in frequency: a review, Eng. Struct. 19 (1997) 718-723, DOI: 10.1016/S0141-0296(96)00149-6

[5] R. Clara Serra, M. Raffy, C. Gontier, A subspace fitting method for structural modal identification in time domain, in: Proceedings of the 25th International Conference on Noise and Vibration engineering (ISMA25), Leuven, Belgium, 2000, https://www.researchgate.net/profile/Rog er_Serra/publication/257297978_A_Subspace_Fitting _ Method For Structure Modal Identification in $\mathrm{Ti}$ me Domain/links/00b495253f3646e6ae000000.pdf\&hl= fr\&sa $=$ X\&scisig $=$ AAGBfm1thGwyXo1CO8tG1vf jIKDP1ZmhGg\&nossl=1\&oi=scholarr\&ved=0ahUKEwi cooK6nOzXAhVGblAKHec2AnIQgAMIKigAMAA.

[6] G. Gautier, R. Serra, J.-M. Mencik, Vibratory diagnosis by finite element model updating and operational modal analysis, Mechanics \& Industry 14 (2013) 145-149, DOI: $10.1051 \% 2 \mathrm{fmeca} \% 2 \mathrm{f} 2013055$

[7] G. Gautier, J.-M. Mencik, R. Serra, A finite element-based subspace fitting approach for structure identification and damage localization, Mech. Sys. Signal Process. 58-59 (2015) 143-159, DOI: 10.1016\%2fj.ymssp.2014.12.003

[8] G. Gautier, R. Serra, J.-M. Mencik Roller bearing monitoring by new subspace-based damage indicator, Shock Vib. (2015) 828093 11, DOI: 10.1155/2015/828093

[9] R. J. Allemang, D.L. Brown, A correlation coefficient for modal vector analysis, in: Proceedings of the 1 st International Modal Analysis Conference \& Exhibit, 1982, pp. 110-116

[10] J.-T. Kim, Y.-S. Ryu, H.-M. Cho, N. Stubbs, Damage identification in beam-type structures: Frequency-based method vs mode-shape-based method, Eng. Struc. 25 (2003) 57-67

[11] N.A.J. Lieven, D. J. Ewins, Spatial Correlation of Mode Shapes: the Coordinate Modal Assurance Criterion (COMAC), in: Proceedings, International Modal Analysis Conference, 690-695, 1988

[12] A.K. Pandey, M. Biswas, M.M. Samman, Damage detection from changes in curvature mode shapes, J. Sound Vib. 145 (1991) 321-332

[13] M. De Roeck, G.A. Wahab, Damage detection in bridges using modal curvatures: application to a real damage scenario, J. Sound Vib. 226 (1999) 217-235, DOI: 10.1006/jsvi.1999.2295

[14] O.S. Salawu, C. Williams, Damage location using vibration mode shapes, in: Proceedings of the 12th International Modal Analysis Conference, Honolulu, Hawaii, USA, 1994, pp. 933939

[15] J.T. Kim, C.R. Farrar, N. Stubbs, Field Verification of a nondestructive damage localization and severity estimation algorithm, in: Proceedings of the 13th International Modal Analysis Conference (IMAC XIII), 182, 1995, pp. 210-218

[16] E. Sazonov, P. Klinkhachorn, Optimal spatial sampling interval for damage detection by curvature or strain energy mode shapes, J. Sound Vib. 285 (2005) 783-801

[17] A.K. Pandey, M. Biswas, Damage detection in structures using changes in flexibility, J. Sound Vib. 169 (1994) 3-17

[18] I. Daubechies, Ten lectures on Wavelets, J. Acoust. Soc. Am. 93 (1993) 1671 
[19] C. Surace, R. Ruotolo, Crack detection of a beam using the wavelet transform, Int. Soc. Opt. Eng. 1994.

[20] K. Liew, Q. Wang, Application of Wavelet Theory for Crack Identification in Structures, J. Eng. Mech. (1998) 152-157

[21] J. Hong, Y.Y. Kim, H.C. Lee, Y.W. Lee, Damage detection using the Lipschitz exponent estimated by the wavelet transform: Applications to vibration modes of a beam, Int. J. Solids Struc. 39 (2002) 1803-1816

[22] E. Douka, S. Loutridis, A. Trochidis, Crack identification in beams using wavelet analysis, Int. J. Solids Stru. 40 (2003) 3557-3569

[23] B. Chen, Y. Kang, P. Li, W. Xie, Detection on structural sudden damage using continuous Wavelet transform and Lipschitz Exponent, Shock Vib. 2015 (2015) 1-17

[24] N. Wu, Q. Wang, Experimental studies on damage detection of beam structures with wavelet transform, Int. J. Eng. Sci. 49 (2011) 253-261

[25] P. Argoul, T.-P. Le, Instantaneous indicators of structural behaviour based on the continuous cauchy wavelet analysis, Mech. Sys. Signal Process. 17 (2003) 243-250, DOI: 10.1006 mssp.2002.1557

[26] G. Venini, P. Naldi, Wavelet analysis of structures: statics, dynamics and damage identification, Meccanica (1997) 223-230

[27] C.-J. Lu, Y.-T. Hsu, Vibration analysis of an inhomogeneous string for damage detection by wavelet transform, Int. J. Mech. Sci. (2002) 745-754

[28] S.G. Mallat, A theory for multi resolution signal decomposition: the wavelet representation, IEEE Trans. Pattern Anal. Mach. Intell. 11 (1989) 674-693

[29] S. Zhong, S.O. Oyadiji, Crack detection in simply supported beams without baseline modal parameters by stationary wavelet transform, Mech. Sys. Signal Process. 21 (2007) $1853-1884$
[30] M. Rucka, K. Wilde, Application of continuous wavelet transform in vibration based damage detection method for beams and plates, J. Sound Vib. 297 (2006) 536-550

[31] M. Masoumi, M.R. Ashory, Damage identification from uniform load surface using continuous and stationary wavelet transforms, Lat. Am. J. Solids Struc. 11 (2014) 738-754

[32] S.G. Mallat, A wavelet tour of signal processing, Academic Press, London, 1999

[33] A.V. Ovanesova, L.E. Suárez, Applications of wavelet transforms to damage detection in frame structures, Eng. Struc. 26 (2004) 39-49

[34] B.K.R. Prasad, N. Lakshmanan, K. Muthumani, et al., Enhancement of damage indicators in wavelet and curvature analysis, Sadhana 31 (2006) 463, DOI: 10.1007/ BF02716787

[35] X. Jiang, Z.J. Ma, W.-X. Ren, Crack detection from the slope of the mode shape using complex continuous Wavelet transform, Comput. Aided Civil Infrastruct. Eng. 27 (2011) 187-201

[36] M. Rucka, Damage detection in beams using Wavelet Transform on higher vibration modes, J. Theor. Appl. Mech. 49 (2011) 399-417

[37] A. Gentile, A. Messina, On the continuous wavelet transforms applied to discrete vibrational data for detecting open cracks in damaged beams, Int. J. Solids Struc. 40 (2003) $295-315$

[38] S. Zhong, S.O. Oyadiji, Detection of cracks in simplysupported beams by continuous wavelet transform of reconstructed modal data, Comput. Struc. 89 (2011) 127148

[39] M. Radzienski, M. Krawczuk, M. Palacz, Improvement of damage detection methods based on experimental modal parameters, Mech. Sys. Signal Process. 25 (2011) 2169-2190

Cite this article as: R. Serra, L. Lopez, Damage detection methodology on beam-like structures based on combined modal Wavelet Transform strategy, Mechanics \& Industry 18, 807 (2017) 